\title{
1 Genomic contacts reveal the control of sister chromosome decatenation in E. coli
}

2 Brenna Conin ${ }^{1,2,3}$, Ingrid Billault-Chaumartin ${ }^{1,4}$, Hafez El Sayyed ${ }^{1,5}$, Charlotte Cockram ${ }^{2, \#}$, Romain

${ }^{1}$ CIRB - Collège de France, CNRS UMR7241, INSERM U1050, PSL University, 11 place Marcelin Berthelot, 75005 Paris, France

${ }^{2}$ Institut Pasteur, Unité Régulation Spatiale des Génomes, CNRS UMR 3525, F-75015 Paris, France

${ }^{3}$ Sorbonne University, Collège doctoral

${ }^{4}$ Present address, Department of Fundamental Microbiology, University of Lausanne Quartier UNILSorge, Biophore Building, CH-1015 Lausanne, SWITZERLAND

${ }^{5}$ Present address, University of Oxford, Biological Physics Research Unit, Clarendon Laboratory,

Department of Physics, Oxford, OX1 3PU, United Kingdom

\# for correspondence:

charlotte.cockram@pasteur.fr

romain.koszul@pasteur.fr

olivier.espeli@college-de-france.fr

\section{Abstract}

In bacteria, chromosome segregation occurs progressively, from the origin to the terminus, a few minutes after the replication of each locus. In-between replication and segregation, sister loci are maintained in an apparent cohesive state by topological links. Whereas topoisomerase IV (Topo IV), the main bacteria decatenase, controls segregation, little is known regarding the influence of the cohesion step on chromosome folding. In this work, we investigated chromosome folding in cells with altered decatenation activities. Within minutes after Topo IV inactivation, a massive chromosome reorganization takes place, associated with increases in trans-contacts between catenated sister chromatids and in long-range cis-contacts between the terminus and distant loci on the genome. A genetic analysis of these signals allowed us to decipher specific roles for Topo IV and Topo III, an accessory decatenase. Moreover we revealed the role of MatP, the terminus macrodomain organizing system and MukB, the E. coli SMC in organizing sister chromatids tied by persistent catenation links . We propose that large-scale conformation changes observed in these conditions reveal a defective 
decatenation hub located in the terminus area. Altogether, our findings support a model of spatial and temporal partition of the tasks required for sister chromosome segregation.

\section{Introduction}

Prokaryotic and eukaryotic chromosomes are not randomly folded, but consist of well-defined structural entities with a complex hierarchical organization. The regulation of this network and its functional interplay with gene expression or other chromosomal metabolic processes such as DNA repair, replication and segregation have been actively investigated in a number of species (Wang and Rudner, 2014). Improvement in imaging techniques of living cells, as well as the development of genomic approaches such as chromosome conformation capture $(3 \mathrm{C} / \mathrm{Hi}-\mathrm{C}$; reviewed in Dekker et al., 2013) techniques have highlighted a mosaic of intertwined structural features including loops, domains, and compartments (Dame et al., 2020).

In bacteria, an important part of genome folding relies on the presence of free DNA supercoils. Independent supercoiling domains were first observed in the 1970's through a combination of molecular biology and electron microscopy approaches (Kavenoff and Bowen, 1976; Sinden and Pettijohn, 1981). These textbook pictures consist of a succession of large ( $200 \mathrm{~kb})$ DNA plectonemes, or microdomains, that are delimited by topological insulators. Genomic and recombination assays confirmed the existence of these microdomains, but found that they are likely smaller in size (10-50 kb), delimited by stochastic barriers (Postow, 2004; Stein et al., 2005). These results suggest that microdomains are not static, and that their size and position can be modulated by DNA transactions such as transcription (Deng et al., 2005) or the binding of proteins to DNA (Leng et al., 2011). The democratization of $3 \mathrm{C} / \mathrm{Hi}-\mathrm{C}$ methodologies provided the opportunity to further assess the impact of DNA supercoiling on chromosome folding. To date, $3 \mathrm{C} / \mathrm{Hi}-\mathrm{C}$ data revealed the organization of large chromosomal features, such as the alignment of replication arms (Le et al., 2013; Marbouty et al., 2015; Wang et al., 2015), macrodomains (Lioy et al., 2018) or inter-chromosomal contacts (Val et al., 2016). At smaller scale, $\mathrm{Hi}-\mathrm{C}$ contact maps of every bacterial genome studied to date display selfinteracting domains (CIDs, 20-200kb) , whose significance remain unclear (Böhm et al., 2020; Le et al., 2013; Lioy et al., 2018; Marbouty et al., 2015; Val et al., 2016; Wang et al., 2015). The boundaries inbetween CIDs correlate with the presence of long, highly expressed genes, or genes coding membrane proteins (Le and Laub, 2016; Le et al., 2013; Lioy et al., 2018).

Simulations suggest that supercoils are able to organize bacterial genome because they would condense DNA and promote the disentanglement of topological domains (Holmes and Cozzarelli, 2000). DNA supercoiling is under tight homeostatic control by topoisomerases. In E. coli, 
Topoisomerase I (Topo I), Topoisomerase III (Topo III), DNA Gyrase and Topoisomerase IV (Topo IV) have all well characterized enzymatic activities (Champoux, 2001; Levine et al., 1998), but understanding the roles played by topoisomerases in chromosomal compaction, folding and organization is still a work in progress. DNA Gyrase, that promotes the formation of free supercoils, is the best candidate for regulation of chromosome organization through supercoiling. $\mathrm{Hi}-\mathrm{C}$ analysis following gyrase inhibition was studied in Caulobacter crescentus, revealing modest changes in chromosome conformation with a slight decrease of 20- to 200-kb contacts that reduced the sharpness and positions of CID boundaries (Le et al., 2013). This observation agrees with recombination data showing that topoisomerase alterations reduce supercoiling domain's size (Staczek and Higgins, 1998). The contribution of other topoisomerases in establishing, maintaining, and regulating genome-wide DNA contacts has, so far, not been investigated.

The E. coli SMC complex MukBEF was shown to be linked to DNA supercoiling, with MukBEF defects being suppressed by Topo I mutation (Sawitzke and Austin, 2000). The suppression correlates with an excess of negative supercoiling promoted by DNA gyrase. In addition, $\mathrm{Hi}-\mathrm{C}$ analysis of a MukB mutant showed an important loss of long-range contacts (Lioy et al., 2018a), suggesting that MukB promotes contacts between distant pairs of loci. One hypothesis is that MukB could promote DNA loops in vivo (Badrinarayanan et al., 2012; Baxter et al., 2019; Carter and Sjögren, 2012; Ruiten and Rowland, 2018). For instance, it is possible that MukB, organised in an axial core, extrudes plectonemic microdomains (Mäkelä and Sherratt, 2020). Interestingly, MukB interacts with ParC, the catalytic subunit of Topo IV. Their interaction changes the catalytic properties of Topo IV (Hayama and Marians, 2010; Li et al., 2010) and modulates its localization (Nicolas et al., 2014; Stracy et al., 2015). In vitro assays suggest that a MukB - Topo IV interaction promotes DNA compaction by forcing the intramolecular knotting activity of Topo IV (Kumar et al., 2017). Single molecule imaging reveals that a small portion of ParC molecules are associated with MukB in small clusters, (Stracy et al., 2015). The absence of MukB leads to a 2 -fold reduction of Topo IV clusters, suggesting that MukB is a driver for the localization of Topo IV in the cell

Topo IV is the main bacterial decatenase. It catalyzes the elimination of precatenanes and catenanes formed during replication (Zechiedrich et al., 1997), and is essential for proper chromosome segregation (Joshi et al., 2013; Kato et al., 1990; Lesterlin et al., 2012; Wang et al., 2008) ( Kato 1990). The requirement of PriA, the main replication fork restart protein, for the survival of Topo IV thermosensitive mutants ( $\operatorname{parE}^{\text {ts }}$ and $\operatorname{parC}^{t s}$ ), suggests that Topo IV inactivation increases replication fork stalling (Grompone et al., 2004). Nevertheless, chromosome replication in parE ${ }^{\text {ts }}$ mutant cells occurs at a similar rate to that seen in WT cells (Wang et al., 2008). Topo IV activity is highest at the dif site positioned in the middle of the Terminus of replication macrodomain, presumably to solve 
catenanes (El Sayyed et al., 2016; Hojgaard et al., 1999; Mercier et al., 2008). However, it also presents hundreds of putative activity sites dispersed on the genome, presumably used for the removal of precatenanes (El Sayyed et al., 2016).

Here we investigate, using $\mathrm{Hi}-\mathrm{C}$, the effect of the different topoisomerases on the $E$. coli chromosome conformation. The inactivation of Topo IV generates the most significant changes in the organization of the chromosome, with a pair of long-range contact patterns, hereafter called butterfly wings, expanding from the terminus macrodomain flanking regions. Imaging experiments confirmed the chromosome reorganization when Topo IV activity is reduced, suggesting that in these conditions distant sister loci segregate when they contact the terminus. Because MatP and MukB also influence the butterfly pattern, we propose that it reveals a particular genome folding dedicated to decatenation. We also showed that when Topo IV is deficient, $\mathrm{Hi}-\mathrm{C}$ reveals inter-molecular contacts between sister chromosomes. Topo III limits these contacts suggesting that it eliminates an important part of precatenanes. Together, our results highlight the ability of $\mathrm{Hi}-\mathrm{C}$ to unveil chromosomal topological features including inter-chromosomal contacts, to improve our understanding of the mechanisms governing chromosome segregation. 


\section{Results}

Topo IV inactivation induces large-scale chromosome conformation changes in enterobacteria

To investigate the respective contribution of each topoisomerase to the genome-wide organization of bacteria chromosomes, we applied capture of chromosome conformation (Hi-C) (Cockram et al., 2021; Lieberman-Aiden et al., 2009); Material and Methods) to exponentially growing Escherichia coli cells either mutated or inactivated for each of the four topoisomerases. The ratio between $w t$ and mutant/depleted cells contact maps were plotted (Material and Methods) (Lioy et al., 2018).

The inactivation of Topo IV using thermosensitive alleles of either the parC (parC $\left.{ }^{t s}\right)$ or parE (parE $\left.{ }^{t s}\right)$ subunits (Kato et al., 1990) resulted in significant changes in the global chromosomal architecture (Figure $1 \mathrm{~A}$ and $1 \mathrm{~B})$. At the permissive temperature $\left(30^{\circ} \mathrm{C}\right)$, the parE $\mathrm{ts}^{\mathrm{ts}}$ mutant grew similarly to wt, with no significant defects in cell growth nor changes in chromosome conformation (Supplementary Figure $\mathbf{1 A}$ and $1 \mathrm{C}$, respectively). Following a 1-hour shift to non-permissive temperature $\left(42^{\circ} \mathrm{C}\right)$, we observed changes in mid- to long-range DNA contacts at three regions of the genome: First, an increase in shortto medium-range contacts from oriC to positions located at $\sim 1 \mathrm{Mb}$ and $\sim 2 \mathrm{Mb}$ on the right and left replichores, respectively. Second, a strong reduction of mid-range contacts in the terminus region (Coordinates 1,315 kb -1,830 Kb, Figure 1A-B) accompanied by an increase of very short-range $(<50 \mathrm{~kb}$, 10 bins) contacts. This particular terminus pattern also featured a strong CID-like border around the dif site. Finally, a peculiar, butterfly-like signal, with two wings of long-range contacts emerges from the flanking regions of the Ter, and extending towards oriC.

These changes in chromosome folding are rescued by a plasmid expressing ParE into the parE ${ }^{\mathrm{ts}}$ mutant cells (Supplementary Figure 1B-1D). Although parC ${ }^{t s}$ mutants are sicker, even at low temperatures (Supplementary Figure 1A), we observed a phenotype similar to that of parE ${ }^{\text {ts }}$, suggesting that the chromosomal changes observed in these mutants are associated with Topo IV inactivation (Figure 1B). To broaden these observations, we applied Hi-C to another y-proteobacteria, Salmonella typhimurium for which thermosensitive Topo IV mutations are available (Luttinger et al., 1991; Springer and Schimd, 1993). Similar conformation changes were observed (Figure 1C and 1D).

Depletion, deletion or inactivation of the three others E. coli topoisomerases (Topo I, Topo III and gyrase) had only mild effect on the global E. coli chromosome organization (Supplementary Figure 1E, 1F and 1G). To reduce Topo I activity we used the topA31 allele (Conter et al., 1997), a topB deletion mutant was used to study Topo III (DiGate and Marians, 1989), and DNA gyrase was studied using a thermosensitive mutant of $g y r B$ (Orr and Staudenbauer, 1981). We observed that although alterations in Topo I and Topo III resulted in a slight loss or gain in short-range contacts, no other significant 
bioRxiv preprint doi: https://doi org/10.1101/2021.05.17 444411 this version posted May 17,2021 . The copyright holder for this preprin (which was not certified by peer review) is the author/funder, who has granted bioRxiv a license to display the preprint in perpetuity. It is made available under aCC-BY-NC-ND 4.0 International license.

changes in the chromosome conformation of these cells were detected (Supplementary Figure 1E and 1F).
A. E.coli Topo IV

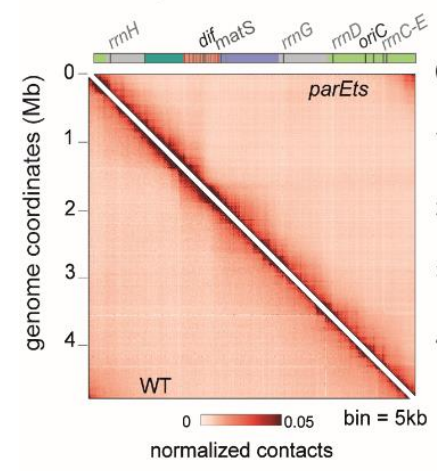

C. S. typhimurium Topo IV

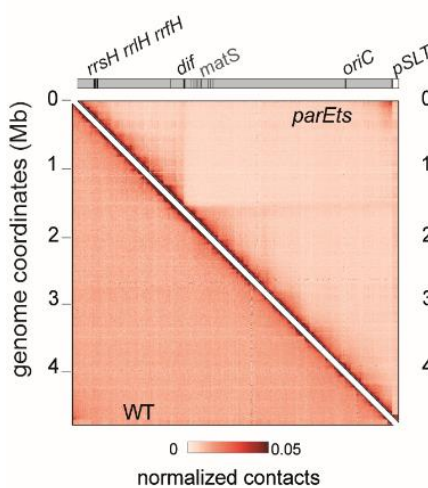

Ratio parEts / WT

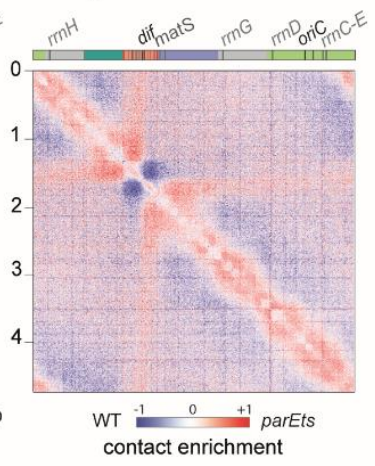

Ratio parEts /WT

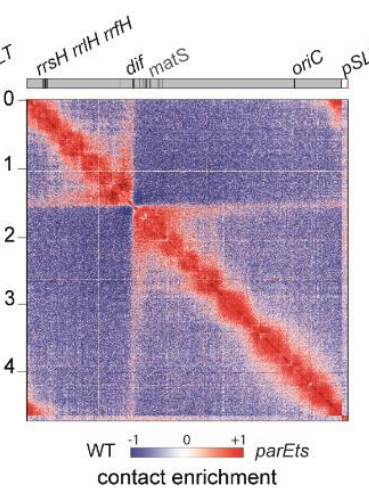

B. E.coli Topo IV

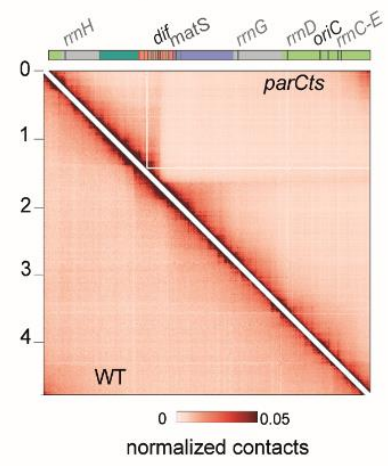

D. S. typhimurium Topo IV

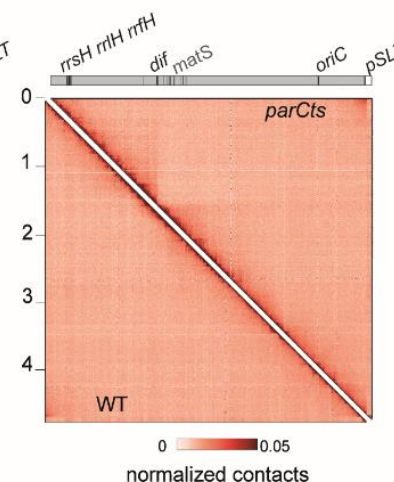

Ratio parCts /WT

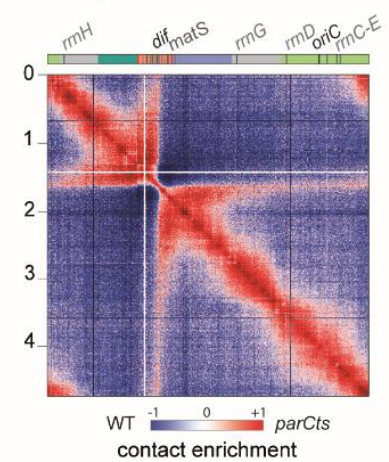

Ratio parCts /WT

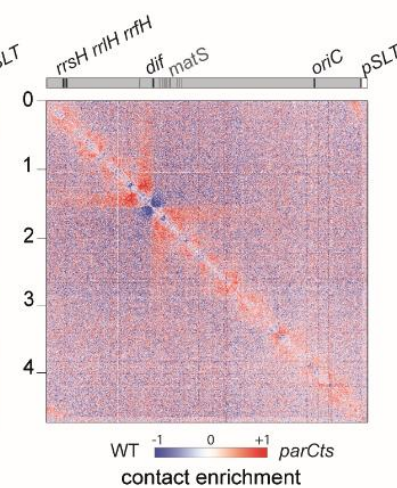

Figure 1: Impact of Topoisomerase IV alteration on nucleoid organization of E. coli and S. typhimurium

For each panels, symmetric halves of the normalized contact map binned at $5 \mathrm{~kb}$ with the wild type (WT) on the bottom and the altered topoisomerase on the top, and the corresponding ratio matrix. (A) parE ${ }^{\text {ts }}$ after $60 \mathrm{~min}$ of shift to non-permissive temperature $\left(42^{\circ} \mathrm{C}\right)$ in $\mathrm{E}$. coli, (B) parC ${ }^{\text {ts }}$ grown at $30^{\circ} \mathrm{C}$ in $\mathrm{E}$. coli, (C) part $E^{\text {ts }}$ after $60 \mathrm{~min}$ of shift to non-permissive temperature $\left(42^{\circ} \mathrm{C}\right)$ in S. typhymurium, (D) parC ${ }^{\text {ts }}$ after $60 \mathrm{~min}$ of shift to non-permissive temperature $\left(42^{\circ} \mathrm{C}\right)$ in S. typhymurium. Genome coordinates are indicated by the $x$ and $y$ axes. Interesting positions of the genome are indicated above the plot. mats sites are represented as grey bars. For E. coli, macrodomains are represented by light green (ori), dark green (right), red (ter), blue (left), gray (NR/NL). For the normalized contact maps, the color scale of the frequency of contacts between two regions of the genome is indicated below (arbitrary units), from white (rare contacts) to dark red (frequent contacts). For the ratio matrices, a decrease or increase in contacts in the mutant cells compared with the control is represented with a blue or red color, respectively. White indicates no differences between the two conditions.

Since gyrase inhibition blocks DNA replication and results in a significant remodeling of the transcriptome (Orr and Staudenbauer, 1981; Peter et al., 2004), gyr $B^{\text {ts }}$ mutant cells were only shifted to the non-permissive temperature for a brief $(20 \mathrm{~min})$ period. Cells exhibit an altered chromosomal 
organization compared to $W T$ cells growing at the same temperature (Supplementary Figure 1G). Inhibition of Gyrase activity resulted in a decrease of short-range contacts. These observations agree with the involvement of gyrase in the definition of supercoiling microdomains or CIDs (Le et al., 2013).

\section{$\mathrm{Hi}-\mathrm{C}$ features observed in parE ${ }^{\text {ts }}$ mutant cells are concomitant with a large reorganization of the nucleoid}

Imaging further supported that nucleoid organization and compaction undergoes a progressive change following parE ${ }^{\text {ts }}$ inactivation. Following the $42^{\circ} \mathrm{C}$ shift, the average DAPI staining density roughly doubled compared to wt (Figure 2A-B). Using parS pMT1 tags in the oriC and terminus region, we analyzed the segregation of sister foci after replication (Figure 2C). In agreement with earlier publications (Wang et al., 2015), we observed a reduction in foci number after 40 min at nonpermissive temperature, suggesting that the increase in nucleoid compaction correlates with the blockage of sister chromatid segregation. In addition, we observed a strong change in the localization of oriC and ter foci within the cell (Figure 2D and 2E and Supplementary Figure 2A and 2B). At permissive temperature, oriC positions preferentially towards the nucleoid edges whereas the ter positions closer to the center of the nucleoid. Upon shift to non-permissive temperature, this organization is flipped, with the ter and oriC migrating towards the cell pole and mid-cell, respectively. These observations suggest that Topo IV inactivation affects both sister chromosome segregation and nucleoid organization.

We then performed a kinetic analysis, using $\mathrm{Hi}-\mathrm{C}$, of chromosome folding after Topo IV inactivation. It allowed us to evaluate whether nucleoid conformation changes correlate with the apparition of new contacts in the Hi-C maps (Figure $2 \mathrm{~F}$ and Supplementary Figure $\mathbf{2 C}$ ). The first consequence of Topo IV inactivation was an immediate loss of short-range contacts over the entire genome (Figure 2F, T10). This drop was gradually substituted by an increase in short- to mid-range contacts, which becomes prevalent along chromosome arms after 40 minutes. Concomitantly the butterfly wings expanded from positions $1 \mathrm{Mb}$ and $2 \mathrm{Mb}$. These features correlated with the evolution of the nucleoid density and organization over the same time period. The terminus region behaved differently, showing a persistent lack of short-range interactions over the entire kinetics. We could not determine whether this terminus pattern was a cause or a consequence of its localization at the nucleoid periphery (Figure 2E). After two hours (t120), we observed similar features with an overall increase of the differences with the wt (Supplementary Figure 2D-G). Altogether, these time course experiments show that specific contacts accumulate at discrete positions surrounding the ter domain upon inactivation of Topo IV, and therefore that Topo IV plays a role in suppressing the formation of long-range contacts within or between sister chromosomes. 
bioRxiv preprint doi: https://doi.org/10.1101/2021.05.17.444411; this version posted May 17, 2021. The copyright holder for this preprint (which was not certified by peer review) is the author/funder, who has granted bioRxiv a license to display the preprint in perpetuity. It is made available under aCC-BY-NC-ND 4.0 International license.

A.

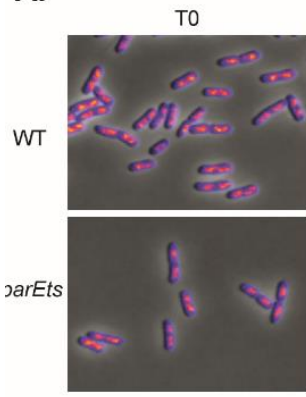

Time point after temperature shift ( $\mathrm{min}$ )
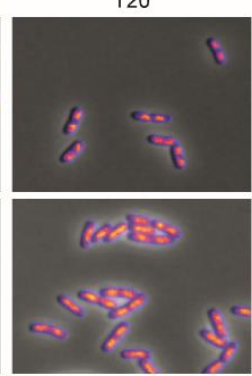

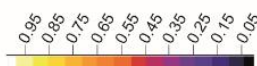

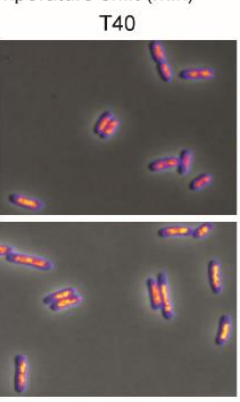

ensity

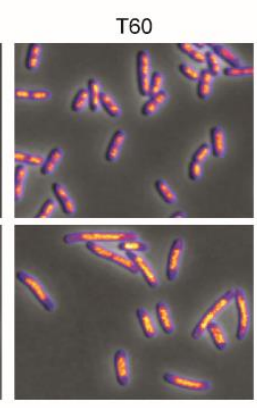

D.
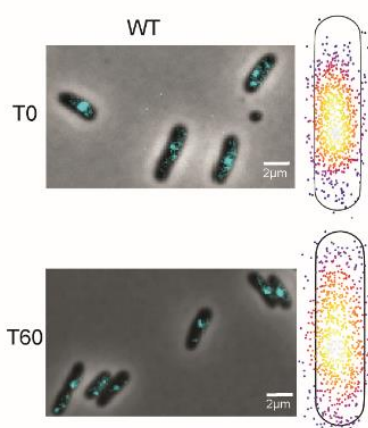

B.

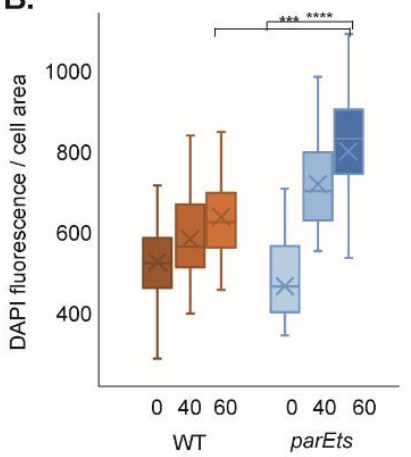

Time after temperature shift (min) c.
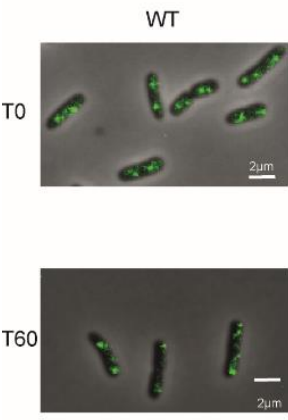
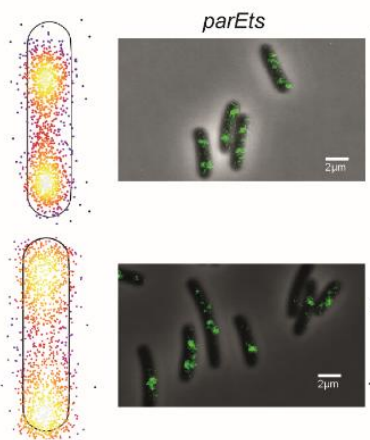
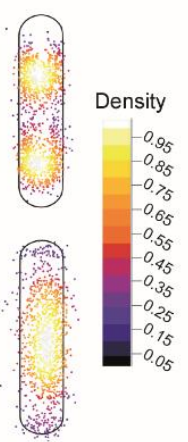

Position of ter foci in the cell

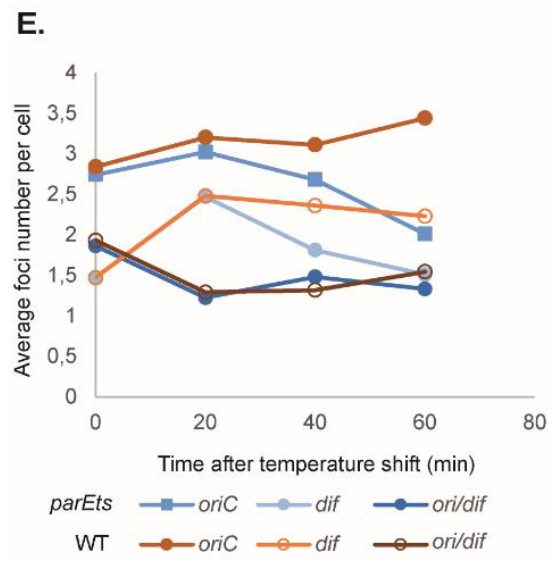

F. Ratio parEts / WT

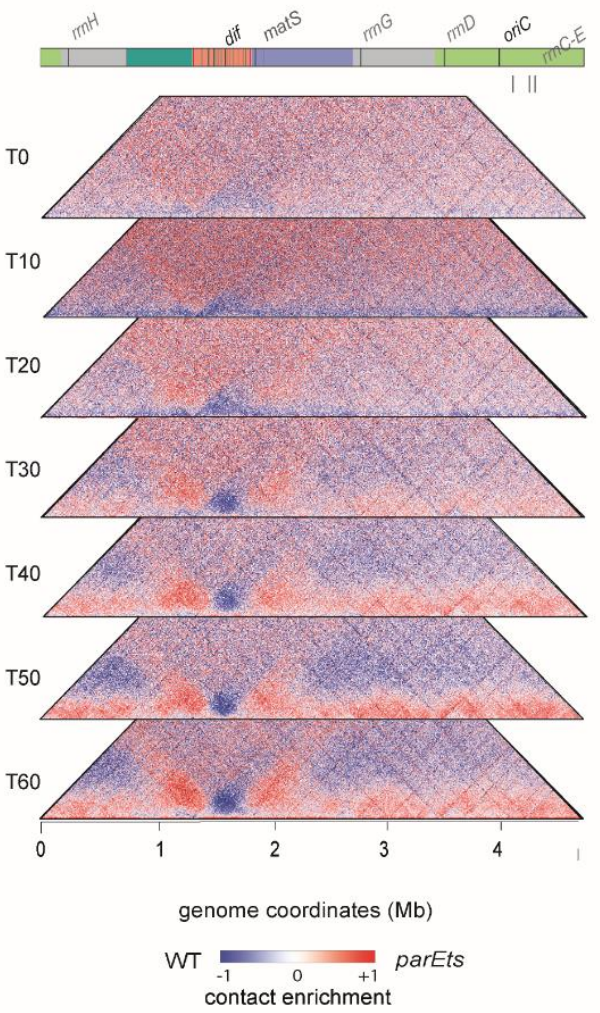


Figure 2: Hi-C features observed in part ${ }^{\text {ts }}$ mutant cells are concomitant with a large reorganization of the nucleoid.

(A) Merged images of phase contrast and DAPI signal (DIC signal in grey and the density of the DNA signal is represented with a fire color scale) of WT (left) and parE ${ }^{\text {ts }}$ (right) cells every 20min during a temperature shift. Scale bar $=2 \mu \mathrm{m}$. (B) Quantification of the fluorescence density as proxy for DNA density across time of the WT (brown boxes) and parE ${ }^{\text {ts }}$ (blue boxes) (a Student test, $\left.{ }^{* * *} P<0.0005\right)$. (CD) Merged images of phase contrast and GFP signal, and average position of the foci in WT (left) and parE ${ }^{\text {ts }}$ (right) cells before temperature shift (TO) and after 60min shift (T60). In (C), GFP signal reveals the position of aidB parS T1 locus (ori) in the cells. In (D), GFP signal reveals the position of fear parS T1 (ter) in the cells. Dots represents each detected focus $(n=200)$ and the color scale represents the density of detected foci in the cell from black (0.05) to white (1). Intermediate time analysis can be found in Supplementary Figure 2A and 2B. (E) Quantification of the number of ori and ter foci across the time course as a proxy for sister chromatids cohesion, and of the ratio ori/ter for WT (brown lines) and parE ${ }^{\text {ts }}$ (blue lines (F) Kinetics of the impact of Topo IV alteration with a time point every 10min after shift at non permissive temperature from to to t60 represented by the ratio matrices of parE $E^{\text {ts }}$ cells versus WT cells kinetics. Normalized contact map of each time point and t120 can be found in Supplementary Figure 2C-F. matS sites are represented as gray bars. Macrodomains are represented by light green (ori), dark green (right), red (ter), blue (left), gray (NR/NL). The y axis indicates the genomic coordinates $(\mathrm{Mb})$. A decrease or increase in contacts in the mutant cells compared with the control is represented with a blue or red colour, respectively. White indicates no differences between the two conditions.

\section{The absence of Topo IV activity results in the co-localization of distant loci with the terminus of the} chromosome

We characterized further the two butterfly-wing stripes emanating at a $45^{\circ}$ angle from the edges of the terminus region in the ratio contact maps between Topo IV and wt (Figure 3A coordinates 1.3 1.5 $\mathrm{Mb}$ and $1.5-1.8 \mathrm{Mb}$, respectively). These signals correspond to an enrichment in medium and longrange contacts by $\sim 200 \mathrm{~kb}$ regions that in one direction span all the way up to oriC, while on the other direction, are blocked by dif, which represents a barrier preventing contacts across each halves of the terminus (Figure 3A and B). Using a $4 \mathrm{C}$-like plot, we investigated how specific $5 \mathrm{~kb}$ bins contribute to the butterfly signal (Figure 3C and D). As illustrated for two representative regions (position 3,010 and $4,440 \mathrm{~Kb}$ ), a small ( $\approx 1.5$ fold), but significant, contact enrichment with the dif zone was detected several megabases away from dif. This enrichment involved several ( 3 to 10 ) bins positioned a few tens of $\mathrm{kb}$ away from dif. The contacts between the dif zone and the 3,010 kb or 4,440 kb regions present a comparable frequency, suggesting that they do not obey to genomic distance law. We observed, on zoomed panels, that the bin containing dif (green dot on Figure 3D) was not involved in the butterfly wing contacts (Figure 3D). To test this exclusion of dif, we measured the sum of contacts made by a $100 \mathrm{~kb}$ window $2 \mathrm{Mb}$ away from dif ( $\Sigma$ sign of Figure $3 \mathrm{~A}$ ) with the $1.2 \mathrm{Mb}$ region containing dif (Figure 3E). This window displayed enriched contacts with most bins from the terminus region over $200 \mathrm{~kb}$ 
241 surrounding dif, but not with dif itself. Their distance independent frequency and the lack of

242 asymmetry at dif strongly suggests that butterfly wings correspond to long-range 3D contacts rather

243 than slithering events.

244 To confirmed the propagation of these very long-range contacts at the single cell level, the inter-focal

245 distances between fluorescently labelled loci positioned at ori and were measured in cells carrying the

246 parC $C^{\text {ts }}$ allele (Figure $3 \mathrm{~F}-\mathrm{I}$ ), that displayed butterfly wings at low temperature $\left(30^{\circ} \mathrm{C}\right)$ (Figure $\mathbf{1 C}$ ). In these

247 conditions, the ori (aidB, 4.4Mb) and the $\operatorname{ter}$ (gusC, 1.69Mb) loci colocalized at the center of the cell in

248 approximately $8 \%$ of cells, a proportion significantly higher than for the wt (Figure $\mathbf{3 G}$ ). The

249 accumulation of the ori region at the center of the cell agrees with the global nucleoid rearrangement

250 observed upon Topo IV inactivation (Figure 2). Ori-ter colocalization was observed close to mid-cell,

251 while most ter foci were localized near the pole of the nucleoid (Figures 2D and $\mathbf{3 H}$ ). Interestingly, the

252 closest distances between sister ori foci were also observed close to mid-cell in the parC ${ }^{t s}$ strain (Figure

253 31). This was in sharp contrast with wt where the minimal distance between sister ori foci was typically

254 observed at the $1 / 4$ and $3 / 4$ positions. This observation suggests that when Topo IV is inactivated, the

255 release of ori pairing takes place closer to mid-cell. Imaging therefore confirms the existence of

256 occasional long-range contacts between the terminus and distant regions of the chromosome in

257 absence of Topo IV activity, and suggests that these contacts may correspond to sister loci segregation

258 attempts. The detection of rare ori-ter colocalization events in wt cells (Figure 3G) suggests that the

259 peculiar chromosome folding events detected in the absence of Topo IV activity might also exist in

260 some wt cells. 
bioRxiv preprint doi: https://doi.org/10.1101/2021.05.17.444411; this version posted May 17, 2021. The copyright holder for this preprint (which was not certified by peer review) is the author/funder, who has granted bioRxiv a license to display the preprint in perpetuity. It is made available under aCC-BY-NC-ND 4.0 International license.

A. Ratio parEts /WT

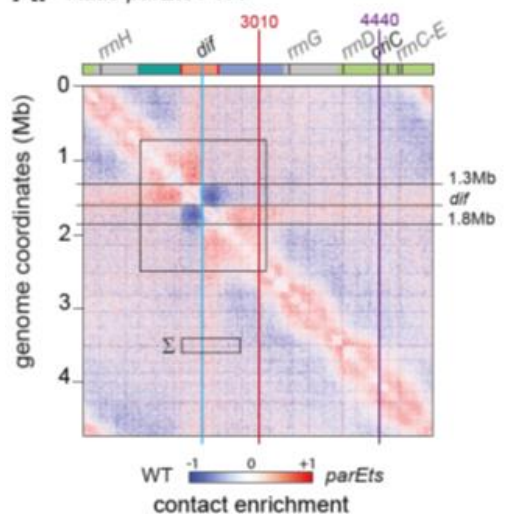

D.

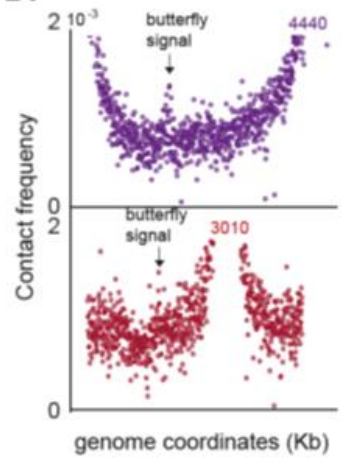

B.

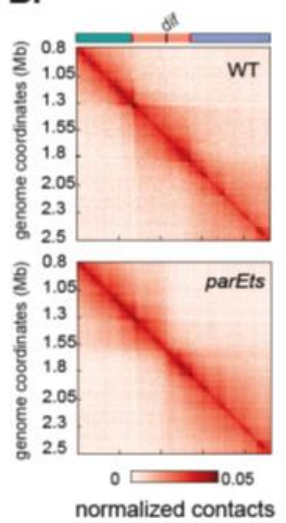

C.

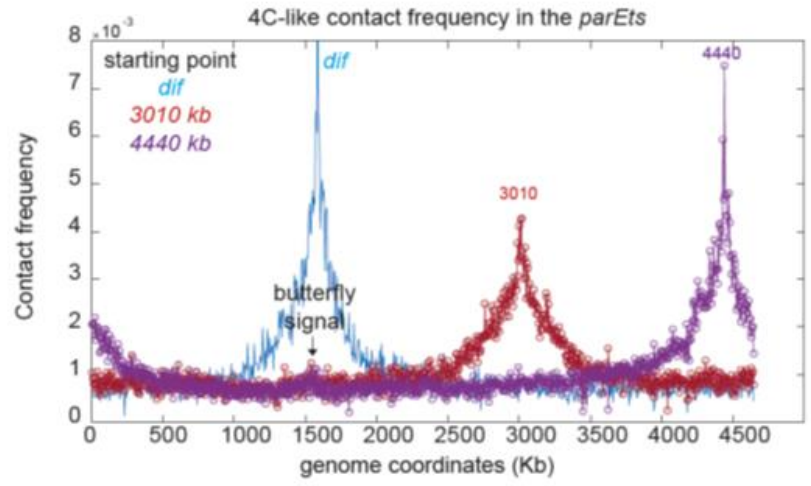

G.

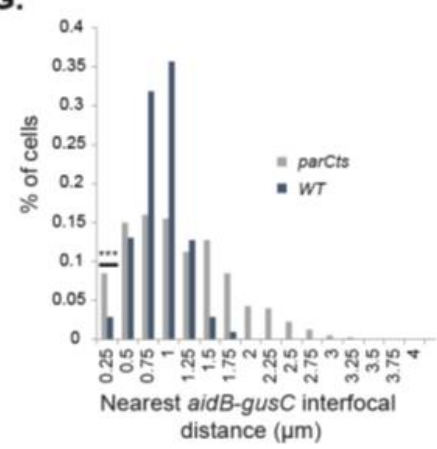

H.

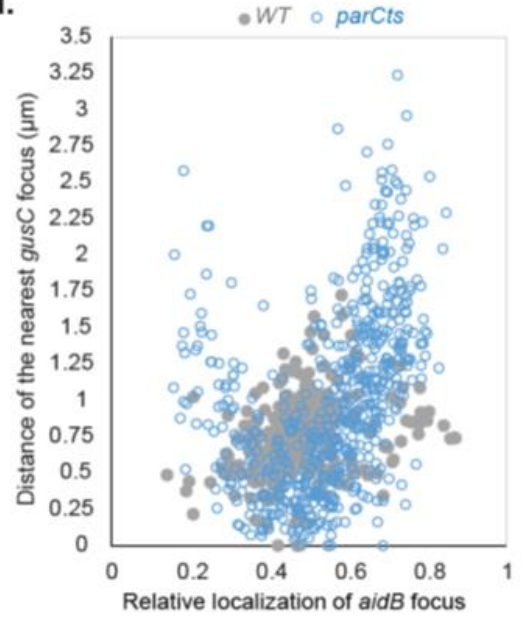

F.

$w T$

gusC parS pMT1 (ter) aidB parS P1 (ori) $\quad 1$ th at $30^{\circ} \mathrm{C}$

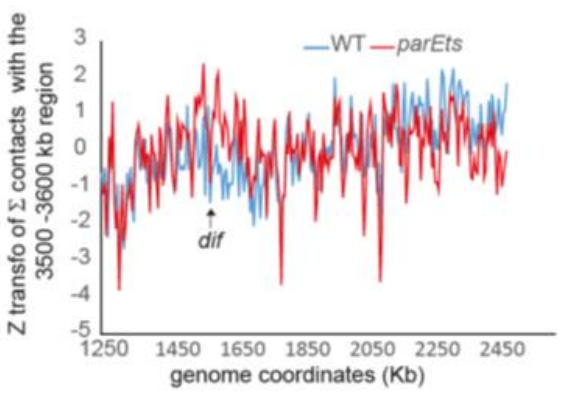

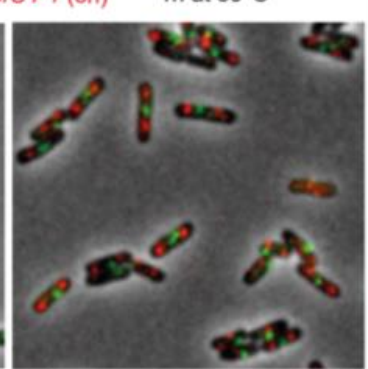

1h at $30^{\circ} \mathrm{C}$

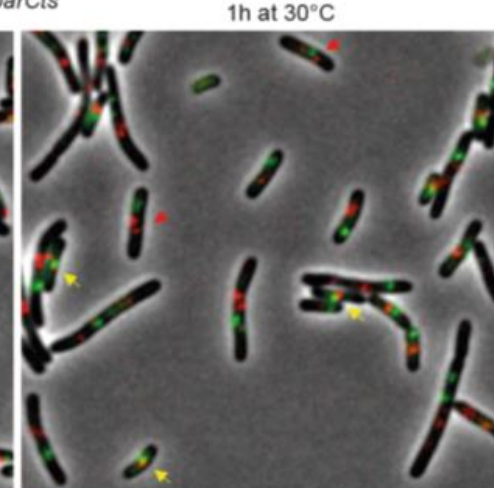

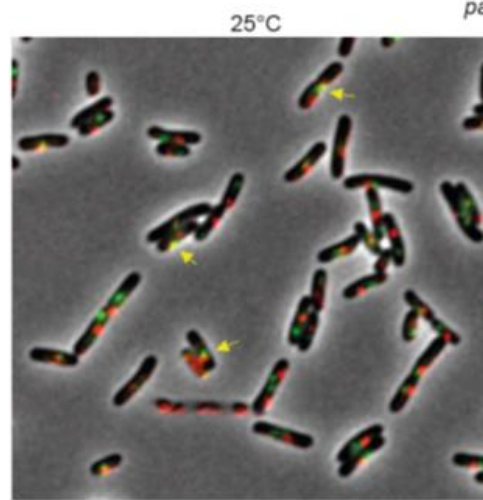

I.

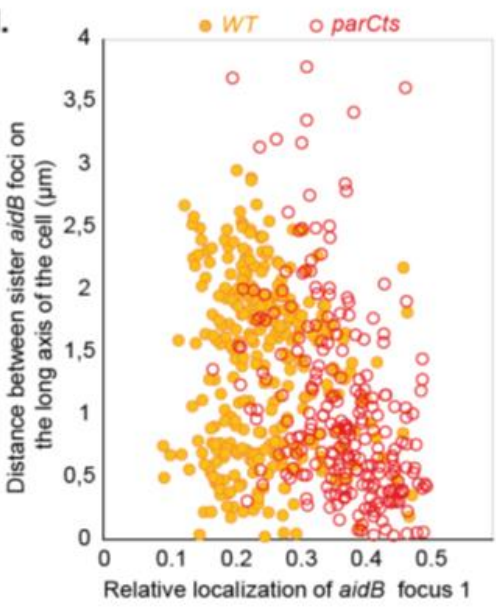


(A) Ratio of the normalized contact map of parE ${ }^{t s}$ and WT where the butterfly wings borders are indicated by gray lines $(1.3 \mathrm{Mb}$ - dif and dif $-1.8 \mathrm{Mb})$ and two representative zones at $3301 \mathrm{~kb}(\mathrm{red})$ and $4440 \mathrm{~kb}$ (purple). (B) Zoom on the terminus macrodomain of the WT and parE $E^{\text {ts }}$ matrices from $0.8 \mathrm{Mb}$ to $2.5 \mathrm{Mb}$ (C) $4 \mathrm{C}$-like plot of contacts from the dif, the 3010 Kb and $4440 \mathrm{~Kb}$ positions, (D) Rescaling of the plot $C$ to show the butterfly wing contacts in the dif area. (E) $Z$ transformation of the contacts made by a $100 \mathrm{~Kb}$ region located between the coordinates $3500 \mathrm{~Kb}$ and $3600 \mathrm{~Kb}$ (marked $\Sigma$ in A) with the terminal part of the genome (coordinates $1250-2450 \mathrm{~Kb}$ ). WT and parE ${ }^{\text {ts }}$ normalized matrix were analyzed. (F) Merged images of phase contrast, CFP and Y-GFP signal. Y-GFP signal reveals the position of gusC parS PMT1 (ter) in the cells and CFP signal reveals the position of aidB parS $P 1$ locus (ori) in the $W T$ (left) and parC ${ }^{\text {ts }}$ cells (right) at permissive temperature $\left(25^{\circ} \mathrm{C}\right.$ ) and after $1 \mathrm{~h}$ at non permissive temperature $\left(30^{\circ} \mathrm{C}\right)$. Yellow arrows indicate colocalisation between aid $B$ and gus $C$ foci and red arrows indicate close sister aidB foci at the center of the cells. Scale bars are $4 \mu \mathrm{m}$. (G) Distribution of the nearest interfocal distance between ter and ori foci in the $W T$ and parC ${ }^{t s}$ strain after $1 \mathrm{~h}$ at $30^{\circ} \mathrm{C}$. $(\mathrm{N}=$ 600 ; colocalization $=$ IFD $<250 \mathrm{~nm}$ ) was analyzed with a KS test, $\left.{ }^{* * *} P<10^{-9}\right)$. (H) Analysis of nearest interfocal distance between ter and ori foci as a function of the relative localization of the ori focus after $1 \mathrm{~h}$ at $30^{\circ} \mathrm{C}$. (I) Analysis of the distance between sister ori foci as a function of the position of the closest ori focus to the pole after $1 \mathrm{~h}$ at $30^{\circ} \mathrm{C}$.

\section{parE $^{\text {ts }}$ inactivation increase inter-chromosomal contacts}

284

285

286

287

288

289

290

291

292

293

294

295

296

297

298

299

300

301

302

The ability of conventional Hi-C to capture intra-chromosomal (cis) contacts is well established. However, its ability to reveal trans contacts between sister-chromatids remains limited given the difficulty to distinguish both homologous molecules without incorporation of modified bases (Oomen et al., 2020). Since Topo IV is mainly known for its involvement in the removal of inter-chromosomal links (or catenanes), presumably between allelic (or near-allelic) loci, we sought to test whether the emergence of prominent $\mathrm{Hi}-\mathrm{C}$ signals spanning throughout the parE ${ }^{\text {ts }}$ contact map could result from trans contacts. We therefore sought to distinguish the contribution of intra vs. inter-molecular contacts to these patterns by determining the impact of DNA replication in absence of Topo IV activity. To do this, we blocked replication initiation by treating WT and parE ${ }^{\text {ts }}$ cells with the amino acid analog dl-serine hydroxamate (Ferullo et al., 2009) (Figure 4A). Following treatment, the cells were then shifted to $42^{\circ} \mathrm{C}$ for 1 hour. Hi-C ratio maps of replicating vs. non-replicating wt cells revealed a significant decrease in mid-range contacts along the entire chromosome in the absence of replication (Figure 4B). Non-replicating parE ${ }^{\text {ts }}$ cells did not show the characteristic pattern observed for replicating cells (Figure 4C and Supplementary Figure 3A-B). Since the stringent response mediated by SHX also reduces transcription of many genes (Sharma and Chatterji, 2010), we tested whether absence of the characteristic pattern in SHX-treated cells could result from a poor transcription. Asynchronously growing parE $E^{\text {ts }}$ and WT cells were therefore treated with the antibiotic rifampicin to inhibit transcription (STAR Methods). Although rifampicin alters Hi-C matrices' quality (Le et al., 2013), the partition of the chromosome into 3 regions in parE ${ }^{\text {ts }}$ cells remained visible upon inactivation of Topo 

presumably because they correspond to inter-chromosome contacts.

A.

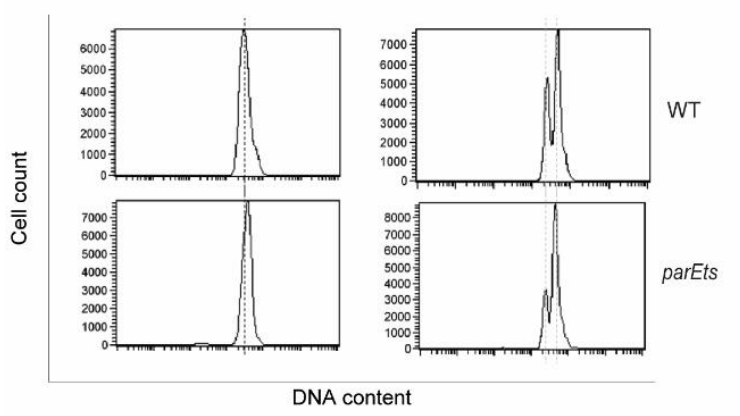

D.

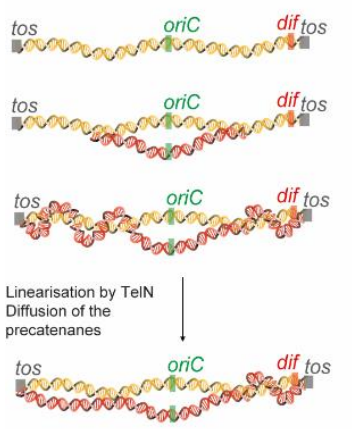

E. Ratio WT linear / WT circular

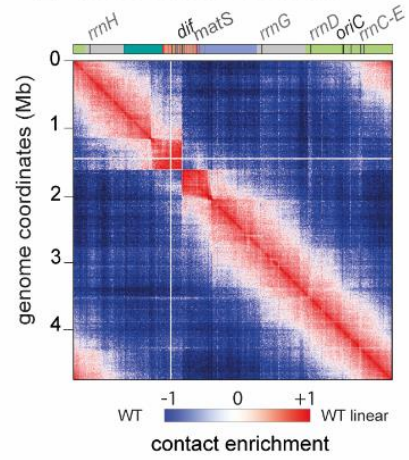

B. Ratio WT + SHX/WT

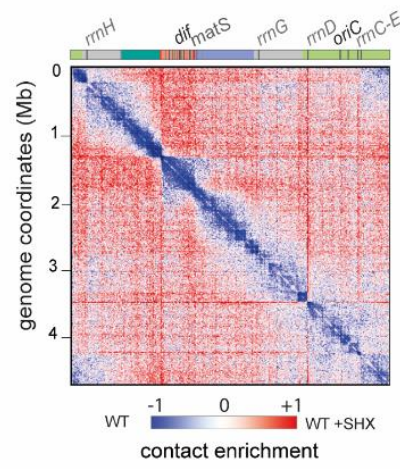

F. Ratio parEts linear /WT linear

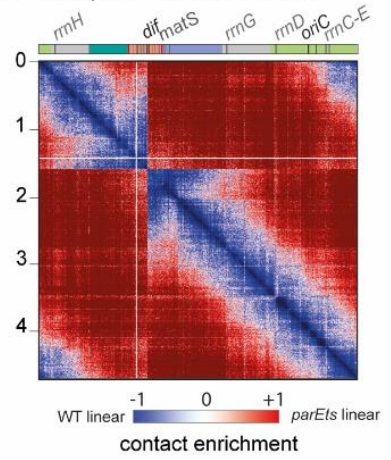

C. Ratio parEts $+\mathrm{SHX} / \mathrm{WT}+\mathrm{SHX}$

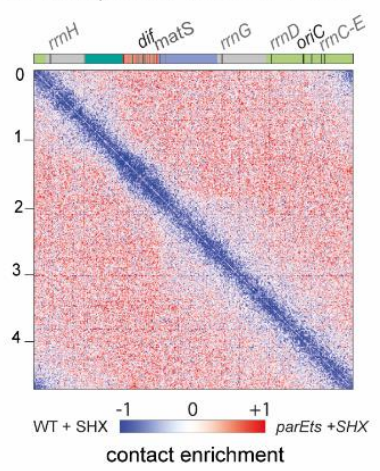

G. Ratio parEts linear / parEts circular

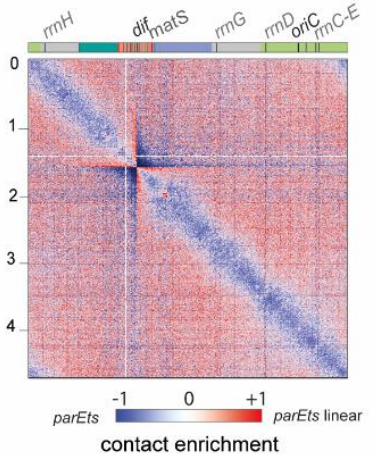

Figure 4: Hi-C features resulting from parE ${ }^{t s}$ alteration result from sister chromosome interactions

(A) FACS of WT and parE $E^{\text {ts }}$ cells after 60 min of shift at $42^{\circ} \mathrm{C}$ untreated (left) and treated with $10 \mathrm{mg} / \mathrm{ml}$ of SHX for 90min total (right). Nucleoid were stained with propidium iodide. Ratio of normalized contacts map binned at $5 \mathrm{~kb}$ of (B) WT + SHX compared to WT, (C) parE ${ }^{\text {ts }}+S H X$ compared to $W T+S H X$. (D) Graphic representation of the replication in E. coli linear strain. The chromosome is linearized via the tos / TelN system of the phage N15. tos sites (gray box) were inserted in the terminus macrodomain. The replication starts at the oriC site (green box) and progress in a bidirectional manner toward the dif site (red box). We hypothesis that precatenanes are formed during the replication of the linear chromosome in the same manner as in the circular one. Sister chromatids are represented by double helices in red (sister A) and yellow (sister B). Before chromosome segregation, the protelomerase TelN linearize the sister chromosomes at the tos site which could possibly allow for diffusion and resolution of the majority of the precatenanes. Ratio of normalized contacts map binned at $5 \mathrm{~kb}$ of (E) linear WT vs circular WT, (F) linear parE ${ }^{\text {ts }}$ vs linear WT, and (G) linear parE ${ }^{\text {ts }}$ linear vs parE $E^{\text {ts }}$ circular. Macrodomains and interesting positions of the genome are indicated above the plot akin to Figure 1. The $y$ axis indicates the genomic coordinates. A decrease or increase in contacts in the mutant cells compared with the control is represented with a blue or red colour, respectively. White indicates no differences between the two conditions. 


\section{Butterfly wings signals are linked to chromosome topology}

328

329

330

331

332

333

334

335

336

337

338

339

340

341

342

343

344

345

346

347

348

349

350

351

352

353

354

355

356

357

358

359

We then wondered whether the circular nature of the E. coli chromosome could lead to topological constraints on the terminus region that would translate into specific contact patterns in the absence of Topo IV. To test this, we performed Hi-C experiments in strains carrying a genome linearized at a tos site, inserted near dif, thanks to the bacteriophage N15 telomerase (Cui et al., 2007). In this strain, Topo IV is still required for growth (Cui et al., 2007). However, it is no longer active at dif, and the filamentation phenotype of the matP mutant is now rescued (El Sayyed et al., 2016). These observations hinted that topological tension resulting from Topo IV inactivation freely diffuses when the N15 telomerase linearizes the duplicated chromosome (Figure 4D). In the wt strain, linearization of the chromosome near dif resulted in an important redistribution of long-range contacts towards short and mid-range contacts (Figure 4E and Supplementary Figure 3E). Upon inactivation of part ${ }^{\text {ts }}$, the Hi-C contact maps of the linear chromosome displayed a significant loss of short- to mid-range contacts, no butterfly wings and no terminus pattern compared to circular wt chromosomes (Figure 4F and Supplementary Figure 3F). The comparison between part ${ }^{t s}$ strains carrying either a linear or a circular genome further revealed a decrease in short/medium contacts along linear chromosomes, in sharp contrast to $w t$ conditions, and compatible with a reduction in the number or density of precatenation links (Figure 4G). This suggests that the linearization of the strain suppresses the accumulation of interminglements between the sister chromatids. Altogether, these observations strongly support the hypothesis that the enhanced mid-range contacts along chromosome arms, as well as the butterfly wings signals, form in response to a failure of Topo IV to remove interchromosomal links.

\section{Topo III activity partially rescues Topo IV deficiencies on chromosome conformation}

Topo III (topB) is a type I topoisomerase whose role during the normal cell cycle remains unclear. Both the modest chromosome conformation changes (Supplementary Figure 1F) and normal viability observed in the mutant (Figure 5A) confirmed that in laboratory growth conditions its role is limited. However, part of its activity is revealed when Topo IV is impaired, as Topo III overexpression rescues parE $^{\text {ts }}$ growth defects (Lee et al., 2019; Nurse et al., 2003; Figure 5A), whereas topB deletion reduces the viability of parE $E^{\text {ts }}$ strains (Figure $\mathbf{5 A}$ ). When parE $E^{\text {ts }}$ was inactivated in a topB deletion strain, the Hi$C$ pattern observed in part ${ }^{\text {ts }}$ mutant cells at the non-permissive temperature was strengthened (Figure 5B), with mid-range contacts increase, and stronger butterfly and terminus patterns (Figure 5B). In the topB parE $E^{\text {ts }}$ strain, long-range contacts were observed between distant regions and a large central region of the ter macrodomain (Figure $\mathbf{5 C}$ ). Their frequency was higher (Figure $\mathbf{5 C}$ ) compared to the 
single parE ${ }^{\text {ts }}$ strain (Figure $3 C$ ). As observed for the parE ${ }^{\text {ts }}$ strain the dif bin appeared to make less longrange contacts than its neighbors (Figure $\mathbf{5 C}$ ). Interestingly, parE $^{\text {ts }}$ features were visible in the contact maps at $30^{\circ} \mathrm{C}$ in the absence of Topo III (Supplementary Figure 4A), suggesting that at this temperature Topo IV is already partially inactivated, but that the organization defects are suppressed by Topo III. This is in good agreement with the CFU data (Figure 5A). Ratio plot and 4C-like analysis also revealed important changes in the distribution of short and mid-range contacts outside of the terminus region when both Topo IV and Topo III are inactivated. When plotting the ratio map between the parE ${ }^{\text {ts }}$ topB matrix and topB or parE ${ }^{t s}$ matrices we characterized the contribution of Topo III and Topo IV. Butterfly wings, and mid-range contacts appeared in the parE ${ }^{\text {ts }}$ topB compared to topB matrices (Supplementary Figure 4B-D). On the other hand, comparing the parE $E^{\text {ts }}$ topB matrix with parE ${ }^{\text {ts }}$ highlighted mostly an increase in short-range contacts (Figure 5D). The overexpression of Topo III rescued the viability of the parE $^{\text {ts }}$ strain at non-permissive temperatures (Nurse et al., 2003, Figure 5A). The Hi-C contact map of a parE ${ }^{\text {ts }}$ strain overexpressing Topo III at non- permissive temperature displayed a reduction in shortrange contacts along the diagonal, but little changes regarding butterfly wings and mid-range contacts (Figure 5E and F and Supplementary Figure 4C and 4D). These observations demonstrate that shortrange contacts are mediated by a topological structure that can be removed by Topo III, most likely precatenanes with single strand regions near the replication fork. Since butterfly wings, mid-range contact and terminus insulation are not suppressed by Topo III overexpression, they appear as a direct consequences of Topo IV inactivation and might therefore reflect the catenation of fully replicated molecules, a substrate impossible to unlink for Topo III (Figure 5G).

The position of butterfly wings is determined by MatP/matS, the Ter macrodomain organizer.

The peculiar positioning of the butterfly wings at the edges of the Ter macrodomain (Figure 3A) prompted us to assess the influence of MatP on these structures. In the absence of MatP and at parE ${ }^{\text {ts }}$ non-permissive temperature, the butterfly wings were replaced by a large region displaying increased long-range contacts (up to $\mathrm{Mb}$ distances), covering the entire terminus macrodomain (Figure 6A). The ratio plot of matP parE ${ }^{t s}$ and matP contact maps further show an enrichment in mid-range contacts within the terminus macrodomain in absence of MatP (Supplementary Figure 5A-D). This observation confirms that the features revealed by Topo IV inactivation are structurally connected, with MatP being responsible for the positioning of the discrete boundaries limiting the entry of putative precatenation links into the terminus macrodomain. These borders define the characteristic butterfly structure and favors the emergence of the terminus pattern. 
A.
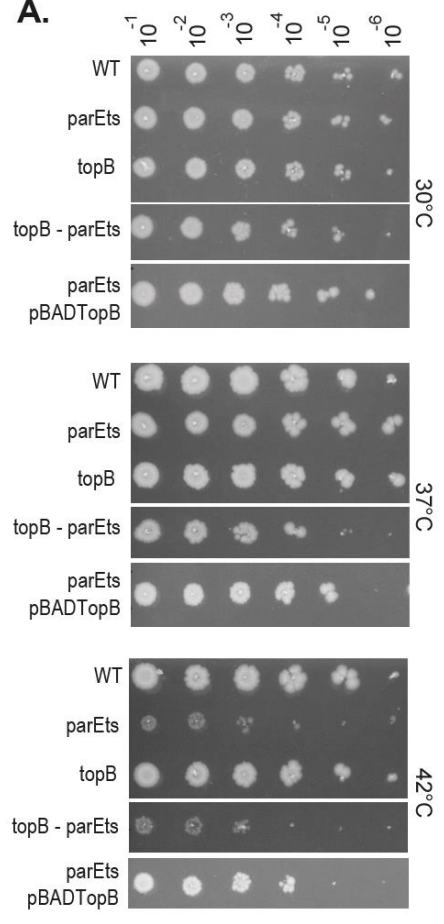

c.

392 from the replication fork.
B.

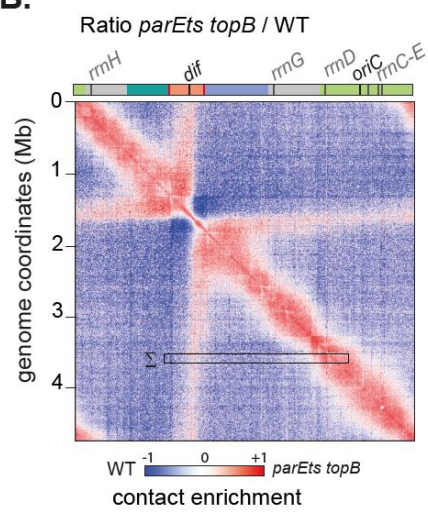

D. Ratio parEts top \& / parEts

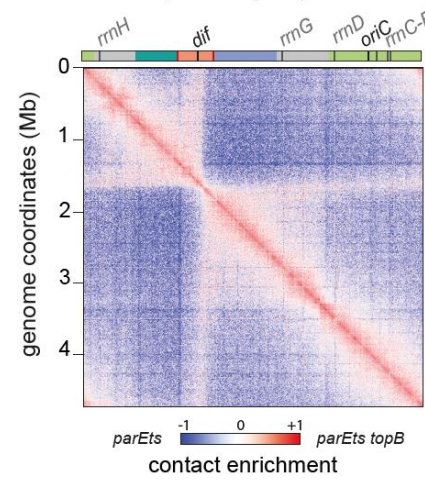

c.

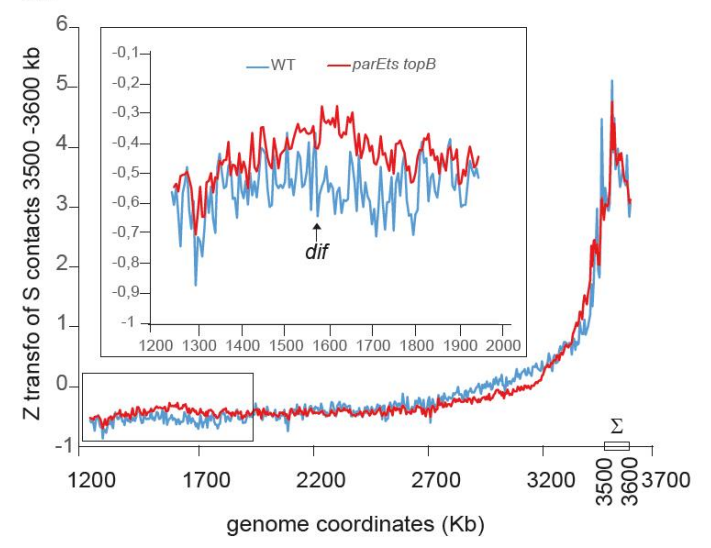

E.

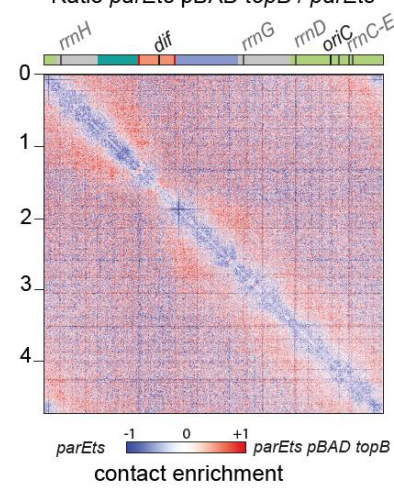

F. Ratio parEts pBAD topB / WT

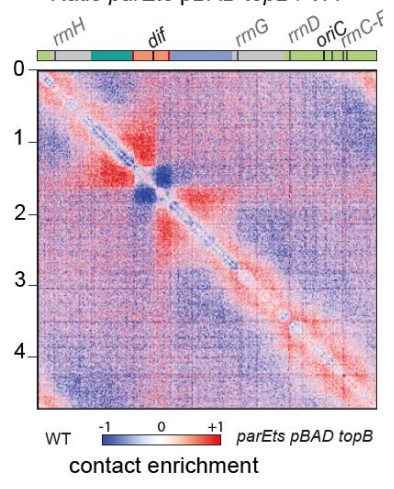

contact enrichment

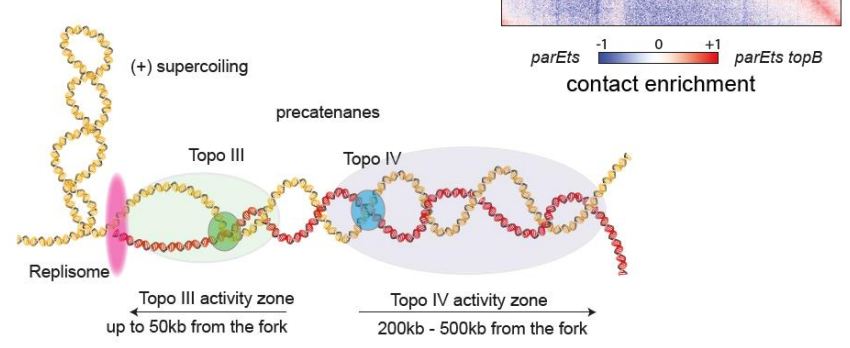

Figure 5: Topo III activity partially rescues Topo IV deficiencies on chromosome conformation

(A) Viability assay performed by droplet CFU of the $W T$, parE $E^{\text {ts }}$, parE $E^{\text {ts }}$ topB, topB, and parE ${ }^{\text {ts }} p B A D$ topB at $30^{\circ} \mathrm{C}, 37^{\circ} \mathrm{C}$ and $42^{\circ} \mathrm{C}$. (B) Ratio of normalized contacts map binned at $5 \mathrm{~kb}$ of for the parE ts topB vs WT matrix. Macrodomains and interesting positions of the genome are indicated above the plot. The $y$ axis indicates the genomic coordinates. A decrease or increase in contacts in the mutant cells compared with the control is represented with a blue or red colour, respectively. White indicates no differences between the two conditions. (C) Z transformation of the contacts made by a $100 \mathrm{~Kb}$ region located between the coordinates $3500 \mathrm{~Kb}$ and $3600 \mathrm{~Kb}$ (marked $\Sigma$ in $\mathrm{B}$ ) with the left replichore (coordinates $1250-3700 \mathrm{~Kb})$. WT and parE ${ }^{\text {ts }}$ topB normalized matrix were analyzed. Inset, zoom on the terminal part of the genome. (D) Ratio of normalized contacts map binned at $5 \mathrm{~kb}$ of for the parE ${ }^{\text {ts }}$ top $B$ vs parE ${ }^{\text {ts }}$. (E) parE ts $p B A D$ topB vs WT. (F) parE ts $p B A D$ topB vs parE ${ }^{t s}$. (G) Graphic representation of the decatenation activity zone of Topo IV (blue) and Topo III (green) after the replication fork (pink). Sister chromatids are represented by double helices in red (sister A) and yellow (sister B). Topo III is able to act directly behind the replication fork at a $50 \mathrm{~kb}$ resolution. Precatenanes beyond the Topo III decatenation activity zone, are dealt with by Topo IV which can act between $200 \mathrm{~kb}$ and $500 \mathrm{~kb}$ away 
Based on these observations, we hypothesized that displacing the terminus macrodomain should reorganize the Topo IV-dependent structural features. We used bacteriophage $\lambda$ site specific recombination to generate an inversion that moved the region associated with the base of the right butterfly wing (coordinate: $1.342702 \mathrm{Mb}$ ) to a region now $540 \mathrm{~Kb}$ upstream the right replichore (coordinate 0. 806 549Mb) (Esnault et al., 2007) (Figure 6B, Supplementary Figure 5E and F). This inversion resulted in the repositioning of five matS sites (matS 1-5) to a region upstream within the right replichore, which in turn resulted in the generation of a large chromosomal region (coordinates $0.8 \mathrm{Mb}$ to $1.3 \mathrm{Mb}$ ) devoid of mats sites and flanked by two mats regions. We observed a shift in the position of the right butterfly wing that coincided with the new position of the inverted matS sites. The butterfly wing was less precise, and long-range contacts appeared to extend from matS5 to dif. The positioning and strength of the second butterfly signal was not affected by the inversion, suggesting that the two butterfly wings are not functionally interlinked (Figure 6B, Supplementary Figure 5E and F).

To test whether the butterfly structure is dynamic, we used an inducible matP gene and followed the formation of structures in the terminus region of the chromosome. After Topo IV inactivation in the absence of MatP, we induced the matP gene using arabinose. Thirty min of induction was sufficient to observe the reestablishment of the terminus pattern and preliminary butterfly wings (Figure 6C, Supplementary Figure $\mathbf{5 G}$ and $\mathbf{H}$ ). These observations suggest that parE ${ }^{\text {ts }}$ characteristic $\mathrm{Hi}-\mathrm{C}$ signals correspond to dynamic structures, that can redistribute upon MatP binding to matS.

\section{MukB controls the shape of the butterfly wings}

The presence of MatP in the terminus macrodomain inhibits MukB activity in WT cells (Nolivos et al., 2016). In the absence of MatP, MukB is able to access the terminus region and has been shown to both accelerate the segregation of terminus loci (Nolivos et al., 2016) and change terminus conformation (Lioy et al., 2018). MukB also interacts with Topo IV and modulate its activity (Hayama and Marians, 2010; Li et al., 2010). We therefore tested whether MukB affects the patterns observed when Topo IV is inactive. A mukB deletion in itself did not create butterfly pattern (Lioy et al., 2018), suggesting that disrupting the ParC-MukB interaction does not alter Topo IV activity in the same way as parE or parC inactivation does. When combined with parE ${ }^{t s}$, the mukB mutation did not abolish the formation of butterfly wings (Figure 6D). However, their characteristics in the double mutant differ from those in the single parE $E^{t s}$ mutant. Comparison of the par $E^{t s}$ mukB mutant with the single parE ${ }^{t s}$ mutant revealed that the basal portion of the butterfly wings were reinforced in the absence of mukB (i.e. higher frequency of contacts) and their length is reduced (Supplementary Figure 5I). In addition, we observed an increase of contacts between all regions within the butterfly, so that they now resemble a 
A. Ratio parEts matP / wild type

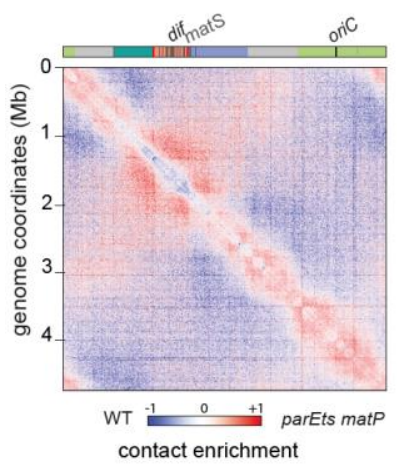

E. Ratio parEts / WT

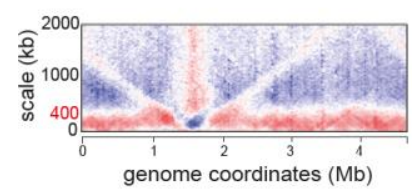

I.

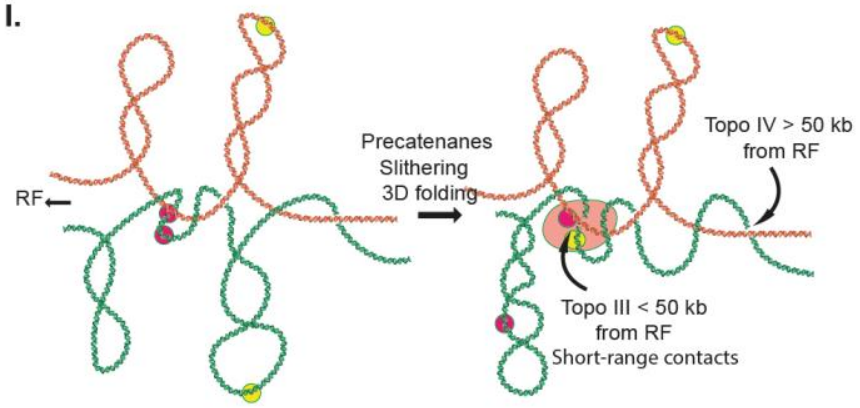
and the bacterial SMC MukB.
B. Ratio parEts inverted / WT inverted

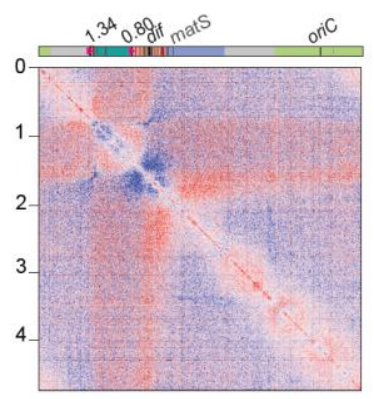

WT inverted ${ }^{-1} \quad 0 \quad+1$ parEts inverted contact enrichment

F. Ratio parEts inverted / WT inverted
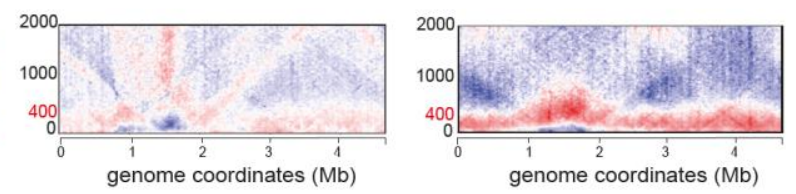

J.
G. Ratio parEts matP / WT

D. Ratio mukB parEts /WT matP parEts
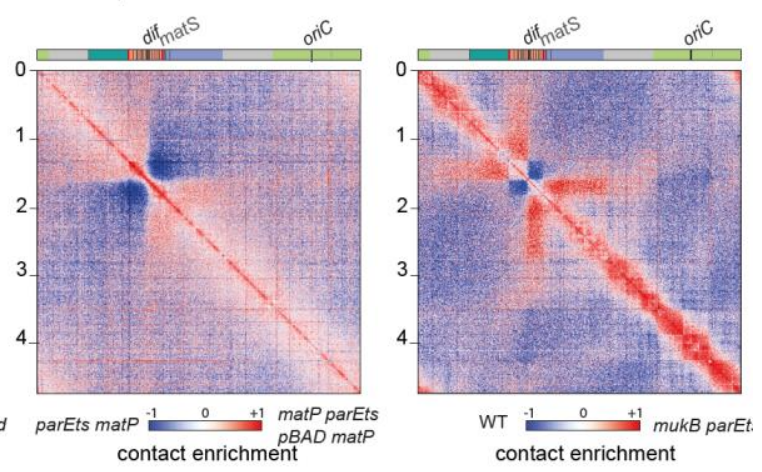

H. Ratio parEts mukB /WT

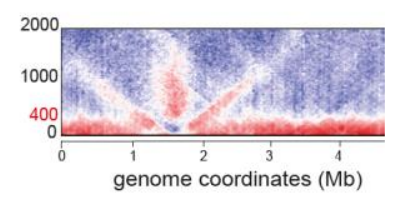

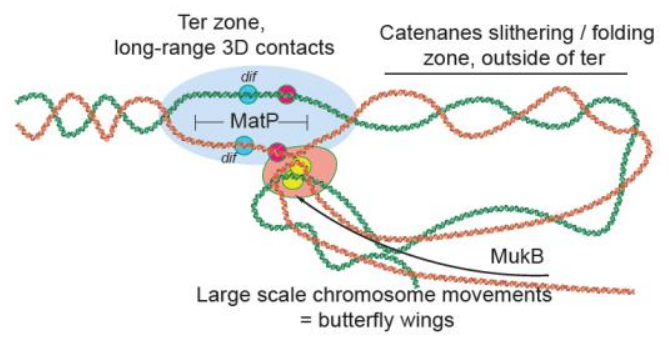

Figure 6: The butterfly position is determined by MatP/matS, the terminus macrodomain organizer

Ratio of normalized contacts map binned at $5 \mathrm{~kb}$, of (A) parE ts matP vs WT, (B) parE $E^{\text {ts }}$ inverted vs WT inverted. The inversion was performed via the attR-attL system and inverted the region between $0.8 \mathrm{Mb}$ and $1.30 \mathrm{Mb}$ resulting in the displacement of matS sites. (C) parE $E^{t s}$ matP pBAD matP vs parE ${ }^{t s}$ matP, (D) parE ${ }^{\text {ts }}$ mukB vs WT. Scalogram ratio of normalized contact map comparing two conditions for each 10$k b$ bin along the chromosome: (E) parE ${ }^{t s} v s W T$, (F) parE ${ }^{t s}$ inverted vs WT inverted and (G) parE $E^{\text {ts }}$ matP vs WT, $(H)$ parE $E^{\text {ts }}$ mukB vs WT. Macrodomains and interesting positions of the genome are indicated above the plot. The $y$ axis indicates the genomic coordinates. A decrease or increase in contacts in the mutant cells compared with the control is represented with a blue or red colour, respectively. White indicates no differences between the two conditions. (I) Graphic representation of putative sister chromatid precatenanes organization, dynamics and homeostasis based on Hi-C data of Topo IV and Topo III mutants. Two pairs of sister loci were represented in pink and yellow respectively. RF stands for replication forks. J) Graphic representation of the terminus region and the chromosome folding events that take place around it when decatenation is impaired. This region may function as a hub where unresolved entanglements of the two sisters formed during chromosome replication enter by a yet unknown mechanism to be separated by Topo IV. The low decatenation capacity in the parE ${ }^{\text {ts }}$ and parC $C^{\text {ts }}$ strains increase the persistence/recurrence of these events and their detection with $\mathrm{Hi}-\mathrm{C}$ and imaging. MukB defines the maximum distance of loci able to contact the decatenation hub. 
self-interacting domain, rather than a stripe (Figure 6D). Scalograms, i.e. the aggregation of contacts over various scales from each bin (Lioy et al. 2018), clearly show the variations in wing shapes and sizes in the mutant strains (parE ${ }^{\text {ts }}$, parE ts $^{\text {inverted, }}$ parE ${ }^{\text {ts }}$ mukB and parE ${ }^{\text {ts }}$ matP) (Figure 6E-H). In the parE ${ }^{\text {ts }}$ strain, butterfly wings extend from the terminus to the origin of the chromosome. In the absence of MatP or in the inverted strain, they nearly disappear. In addition, MatP prevents the formation of midrange contacts in the terminus region (Figure 6F). Finally, in the absence of MukB the butterfly wings abruptly stop 1 Mb from dif on both replichores. The absence of MukB results in the exclusion of the oric region from the butterfly structure. The increase in short range contacts detected along chromosome arms when Topo IV is inactivated (presumably corresponding to the accumulation of precatenanes) did not change in the absence of matP or mukB. This suggests that Topo IV partners do not significantly impact precatenanes dynamics. Therefore, MatP is involved in the positioning of the butterfly pattern, while MukB determines the wing length and contact density (Figure 6H). Moreover, MatP itself and/or the resulting MukB relocalization might prevents the progression of precatenanes into the terminus (Figure 6F).

\section{Discussion}

\section{Alteration of each Topoisomerase induces specific chromosome conformations}

Although DNA topology plays an important role in bacterial chromosome folding and compaction, the consequence of topoisomerase alteration on these processes are not yet fully understood. We took the advantage of $\mathrm{Hi}-\mathrm{C}$ protocol improvements in bacteria to analyze chromosome conformation upon alteration of each of the four Topoisomerase of E. coli. Inhibition of Gyrase activity results in a strong reduction of short-range contacts over the entire genome. This agrees with previous report (Le et al., 2013) and confirms that supercoiling homeostasis is an important driver of CID organization in bacterial genomes. Surprisingly, because they have opposite catalytic activities, Topo I inhibition also reduces short-range contacts. Topo III and Topo IV inhibitions have the opposite effects, as they both increase short-range contacts: lightly and homogeneously for Topo III, but much more significantly and with a robust patterning for Topo IV. We investigated in details the nature and determinants of this patterning.

\section{New chromosome contacts emerging from Topo IV inhibition are intermolecular contacts}

Topo IV inhibition induces an increase of chromosome contacts in the 50 - $200 \mathrm{~kb}$ range chromosome arms with the exception of a large terminus region that behaves differently. We observed that these 
new contacts are only established in replicating cells carrying circular chromosomes. Moreover, the activity of Topo III, which appears at very short range $(0-50 \mathrm{~kb})$, limits their amount. Altogether, these results demonstrate that short - mid range contacts detected when Topo IV is inactivated are produced by precatenanes (Figure 6I). Their amplitude, 200-300 kb, can only be explained if catenated sister chromatids slides or fold in 3D on top of each other on a 200-300 kb window. By contrast, Topo III only modulated short scale contacts, which agrees with the idea that soon after replication sister loci are well aligned (no more than 50kb offset). In the absence of Topo IV some of these precatenane links persist away from the replication fork, out of reach of Topo III action even when overexpressed. Therefore, a few tens of second after their replication, catenane links cannot reach the Topo III activity zone, and might be entrapped by topological barriers (Figure 6I). The comparison of wild-type replicating and non-replicating cells (Figure 4B) confirms that during a regular cell cycle Topo IV and Topo III sporadically let few catenation links all over the genome that will contribute to sister chromatid cohesion (Joshi et al., 2013; Lesterlin et al., 2012). Future Hi-C and sisterC (Oomen et al., 2020) experiments performed on cells with synchronized cell cycle should give important insights on the nature of these inter-sister contacts and their molecular determinants.

\section{Very long-range contacts emerged as a butterfly pattern at the terminus}

The butterfly pattern observed in the absence of Topo IV is an atypical Hi-C feature; Butterfly wings involve contact between the dif area (200 kb surrounding dif) and the rest of the chromosome. These contacts don't follow genomic distance law and are not impacted by cis barriers (Figure $\mathbf{3}$ ). The density of contacts inside butterfly wings is dependent on the topological status of the cell (Topo III effect) and on MukB and their position determined by MatP/matS (Figure 6). We propose that the butterfly pattern is the signature of a failing decatenation hub (Figure 6J). dif is the major Topo IV activity site during regular cell cycle, this suggests that a large number of catenation links are removed at this locus (El Sayyed et al., 2016; Hojgaard et al., 1999). By contrast, the rest of the terminus region presents few Topo IV activity sites, but two prominent Topo IV binding sites at 1.2 and $2.8 \mathrm{Mb}$ (El Sayyed et al., 2016). The positions of these binding sites corresponds to the border of the butterfly wing bases. Butterfly wings correspond to 3D contacts between the dif area and the rest of the chromosome. However, contacts between distal butterfly wings' regions were not enhanced (Figure 3). Since butterfly wings were more robust when Topo III was inhibited and less robust when Topo III was overexpressed, we postulate that they correspond to contacts between catenation links scattered over the chromosome arms and the dif area (Figure 6J). Imaging confirmed the possibility of such contacts between the ori and terminus region in a small portion of the cells in a population, respectively $8 \%$ in parE $^{\text {ts }}$ and $2 \%$ in wt cells (Figure 3). Several mechanisms might drive these contacts. One possibility is that protein marking catenation links is involved in protein-protein contacts between dif and other 
regions of the chromosome. A DNA binding protein marking positive supercoils, GapR, has been identified in C. crescentus (Guo et al., 2018). A yet unknown equivalent of GapR for catenation links (catenation crossing are topologically similar to positive supercoils) might exist in E. coli. Future experiments will be require to test if YejK which interacts with Topo IV and binds DNA (Lee and Marians, 2013) or another catenane binding factor, could play such a role. Another possible explanation is that regions of the chromosome presenting excess of catenation links are excluded from the bulk of the genome and are then available to interact with the decatenation hub in the terminus. Since the absence of MukB changes butterfly wing density, one hypothesis is that catenane links cannot be extruded as plectonemic loops. Successive unsuccessful contacts between a crippled decatenation hub in the terminus area and links dispersed on the genome might create the butterfly pattern (Figure 6J). The role of such contacts for chromosome segregation remains unknown, however the observation of duplicated ori foci close to the terminus foci and close to mid-cell in the parc ${ }^{\text {ts }}$ strain (Figure $\mathbf{3 N}$ ) suggest that this localization may promote decatenation perhaps by concentrating the few remaining active Topo IV in a hub like structure.

\section{Chromosome conformation capture proposes a new read out of topological constraints}

Most of our knowledge on the control of topological homeostasis by topoisomerases comes from plasmid DNA topology analyses by 1D or 2D gel electrophoresis or electron or AFM microscopy (Cebrián et al., 2015). Recently, molecular genomics methods were implemented to map topoisomerase activities or binding on chromosome (McKie et al., 2020). Hi-C is a popular method to study chromosome conformation, and a derivative to track intersister contacts was recently designed in eukaryotes (Oomen et al., 2020). The use of loxP recombination to reveal proximity of sisters (Lesterlin et al., 2012) or their catenation (Mariezcurrena and Uhlmann, 2017) is also a powerful tool to decipher local chromosome topology, with a genome-wide loxP recombination based assay recently adapted to survey sister proximity in $V$. cholera (Espinosa et al., 2019). Our results suggest that Hi-C can also offer a read-out of topological constraints. Coupling all these approaches together, along with imaging, will refine our understanding of chromosome conformation dynamics during the bacteria cell cycle and particularly the contribution of inter-sister contacts. 
Strains: The strains used for this study can be found in Supplementary table 1. All strains are derived from MG1655. All strains were grown in minimal media A (0.26M KH2PO4, 0. 06M K2HPO4, 0.01M tri sodium citrate, $2 \mathrm{mM}$ MgSO4, $0.04 \mathrm{M}$ (NH4)2 SO4) supplemented with $0.2 \%$ of casamino acids and $0.5 \%$ of glucose. All strains were grown at $30^{\circ} \mathrm{C}$. The strain containing the thermosensitive allele of gyrB $\left(\right.$ gyrB $B^{\text {ts }}$ ) was then shifted at $42^{\circ} \mathrm{C}$ for $20 \mathrm{~min}$; the strain containing the thermosensitive allele of parC $\left(p a r C^{t s}\right)$ grown at $30^{\circ} \mathrm{C}$ which is already non-permissive temperature; and, the strain containing the thermosensitive allele of parE ( $\operatorname{parE}^{t s}$ ) was shifted at $42^{\circ} \mathrm{C}$ for $60 \mathrm{~min}$ at most. Time course of parE $E^{\text {ts }}$ shift was performed by growing the cells at $30^{\circ} \mathrm{C}$ before performing a shift for $10,20,30,40,50$ and 60 min. Strains containing the expression plasmid $\mathrm{PBAD}$ were cultivated with $0.2 \%$ arabinose to induce the expression of the gene under the control of the promoter pBAD. For the strain containing pBAD topB, the arabinose was present for the entire duration of the culture; for the strain containing pBAD matP, arabinose was added after $30 \mathrm{~min}$ of shift to $42^{\circ} \mathrm{C}$.

Drugs and antibiotics: Inhibition of Topo I was done with a $5 \mathrm{~min}$ treatment with $50 \mathrm{ng} / \mu \mathrm{l}$ of Topetecan (Subramanian et al., 1995). DL-Serine Hydroxymate (SHX, Sigma CAS Number 55779-32-3), an inhibitor of seryl-tRNA synthetase which triggers the stringent response and prevents new rounds of replication, was used at a $10 \mathrm{mg} / \mathrm{ml}$ working concentration for $90 \mathrm{~min}$. The efficiency of the drug is checked by FACS. Rifampicin was used for $10 \mathrm{~min}$ at a $100 \mathrm{ng} / \mu \mathrm{l}$ working concentration to inhibit transcription.

Fluorescence activated cell sorting analysis: The number of nucleoid was monitored using BD fortessa cytometer with a $488 \mathrm{~nm}$ argon laser and 515-545 nm emission filter at a maximum of 5000 event per second. A minimum of 100000 cells were analyzed per time point. Calibration was done with the samples of the stationary phase of the $W T$ and parEts at $30^{\circ} \mathrm{C}$. For all samples, approximately $10^{8}$ cells were fixed in $70 \% \mathrm{EtOH}$, washed, marked with propidium iodide $(2-20 \mu \mathrm{g} / \mathrm{ml})$, washed again, and resuspended in sterile 1× PBS pH 7.2 prior to analysis. FCSalyzer software (https://sourceforge.net/projects/fcsalyzer/) was used for data analysis.

Hi-C libraries: $\mathrm{Hi}-\mathrm{C}$ libraries were generated as recently described in (Cockram et al., 2021). $30 \mathrm{~mL}$ of culture was grown in Minimal Medium A supplemented with casaminoacids and glucose until OD600nm 0.2. Protein-DNA interactions were cross-linked by the addition of $37 \%$ formaldehyde ( $3 \%$ final concentration) for $30 \mathrm{~min}$ at room temperature with gentle agitation. Crosslinking was quenched with $2.5 \mathrm{M}$ glycine ( $0.4 \mathrm{M}$ final concentration) for $20 \mathrm{~min}$ at room temperature with gentle agitation. Fixed cells were then collected by centrifugation $\left(4000 \times \mathrm{g}, 10 \mathrm{~min} 4^{\circ} \mathrm{C}\right)$, washed once in $1 \times \mathrm{PBS}$ and snap frozen on dry ice and stored at $-80^{\circ} \mathrm{C}$ until use. To proceed to the digestion of the cells, pellets were thawed on ice and resuspended in $1.2 \mathrm{~mL}$ of $1 \mathrm{xTE}+$ complete protease inhibitor (EDTA-free, 
591 Roche). Cells were transferred to a VK05 tubes containing glass spreads, and then lysed with precellys

592 (V750; 5x30s). Uncross linked proteins were then solubilized by incubating the lysed cells with $10 \%$ SDS

$593(0.5 \%$ final) for $10 \mathrm{~min}$ at room temperature. $1 \mathrm{ml}$ of lysed cells was then added to the digestion mix

$594(3 \mathrm{ml}$ of $\mathrm{dH} 2 \mathrm{O}, 0.5 \mathrm{ml}$ of $10 x$ NEBuffer $1,0.5 \mathrm{ml}$ of $10 \%$ triton-X100, and $1000 \mathrm{U}$ Hpall) and incubated $3 \mathrm{~h}$

595 at $37^{\circ} \mathrm{C}$. Digested cells were pelleted by centrifugation and resuspended in $398 \mu$ l of water before being 596 added to the biotinylation mix (10X ligation buffer without ATP; dAGTtp 3.3mM; biotine-14-dCtp

$5970.4 \mathrm{mM} ; 50 \mathrm{U}$ klenow $(\mathrm{NEB} 5 \mathrm{U} / \mu \mathrm{I})$ ) and incubated $45 \mathrm{~min}$ at $37^{\circ} \mathrm{C}$ with agitation. Ligation mix (10X ligation

598 buffer without ATP; BSA 10mg/ml; ATP 100mM; 250U thermoFisher T4 DNA ligase) was then added to

599 the biotinylated DNA and the mix was incubated $3 \mathrm{~h}$ at room temperature with gentle agitation.

600 Protein-DNA complexes were then reverse crosslinked by adding the $20 \mu$ l of EDTA $0.5 \mathrm{M}, 80 \mu \mathrm{l}$ of SDS

$60110 \%$ and and $100 \mu \mathrm{l}$ of proteinase $\mathrm{K} 20 \mathrm{mg} / \mathrm{ml}$ and incubating at $65^{\circ} \mathrm{C}$ overnight. DNA extraction is made

602 by phenol- chlorophorm and precipitation with ethanol. DNA is washed with $70 \%$ ethanol,

603 resuspended in $130 \mu \mathrm{l}$ of TE and then treated with $20 \mathrm{mg} / \mathrm{ml}$ RNAse for $30 \mathrm{~min}$ at $37^{\circ} \mathrm{C}$. Samples were

604 sonicated using a Covaris S220 instrument to obtain fragments between $300 \mathrm{bp}-500 \mathrm{bp}$, then purified

605 by AMPure XP beads. The Illumina process was performed according to manufacturer

606 recommendation, with are 12 cycles of amplification. The size of the DNA fragment in the libraries are

607 checked on TAE 1\% agarose gel and subjected to paired-end sequencing on an Illumina sequencer

608 (NextSeq500-550 - 75 cycles).

609 Generation of contact maps: Generation of contact maps was done with E.coli analysis pipeline developed by Axel Cournac (https://github.com/axelcournac/EColi analysis). Briefly, reads recovered from the sequencing were aligned with bowtie2 on the reference E. coli genome (NC_000913), the two ends were merged and the reads were filtered to have a mapping quality strictly $>30$. Then each read was assigned to a Hpall restriction fragment (fragment attribution), more than $80 \%$ of the reads on average are conserved as informative reads. The contact matrices files are then generated, binned at 2,5 or $10 \mathrm{~kb}$ and normalized through the sequential component normalization procedure (SCN (Cournac et al., 2012)).

Ratio and Ratio plots: In order to visualize the differences between two contact maps, their ratio is calculated as described in (Lioy et al., 2018). Briefly, contacts made in the mutant map are divided by the contacts made in the control map for each loci. Increase of decrease of contact in the mutant compared to the control will appear red or blue respectively and no changes will appear white. To visualize the contact signal at a smaller scale we use the ratio plot representation, as described by (Lioy et al., 2018). Briefly, the ratio-plots summarize the differences of contacts between two conditions made by each bin with its neighboring bins, regardless of their orientation. For each condition, the 
distances (from $5 \mathrm{~kb}$ to $1000 \mathrm{~kb}$ or to $2000 \mathrm{~kb}$, in $5 \mathrm{~kb}$ increments), was computed The $\log 2(\mathrm{ACmutant} / \mathrm{ACwt}$ ) was then displayed as a heatmap.

Viability assay: For each strains, cells were serially diluted in LB (10-1 to 10-8) and $1 \mu$ l of each dilution is deposed on LB agar plates. Plates were incubated overnight at $30^{\circ} \mathrm{C}$.

DNA density analysis: Cells were grown using the same conditions as $\mathrm{Hi}-\mathrm{C} .1 \mathrm{ml}$ of culture of a control sample at $30^{\circ} \mathrm{C}$ and a sample after a $60 \mathrm{~min}$ shift at $42^{\circ} \mathrm{C}$ for each strain were fixed every $20 \mathrm{~min}$ (tot60), using an equal volume of 1X PFA (37\% paraformaldehyde + glutaraldehyde $+1 X$ PBS). DAPI $(0.5 \mu \mathrm{g} / \mathrm{ml})$ is added and the mixture is incubated $10 \mathrm{~min}$ in the dark. Cells were then washed and deposited on microscopy slides containing a freshly made agarose pas (1X PBS $+1 \%$ agarose). Samples were observed with a Spinning disk (YokogaWa) W1 system on a Zeiss inverted confocal microscope with a 63x, phase objective. To avoid bleaching, each field of view was only observed in the phase contrast channel before acquisition. Using ImageJ, the density of DAPI fluorescence in each nucleoid was calculated as a proxy for nucleoid density.

Loci positioning analysis: Cells were grown akin to $\mathrm{Hi}-\mathrm{C}$ samples (above). $1 \mathrm{ml}$ of culture of a control at $30^{\circ} \mathrm{C}$ and a sample after shift at $42^{\circ} \mathrm{C}$ for each strain, were fixed every $20 \mathrm{~min}$ (t0-t60), using an equal volume of fixation medium (3.7\% formaldehyde, 0,006 \%glutaraldehyde, 1X PBS). Cells were pelleted and resuspended in $100 \mu \mathrm{l}$ of fresh Minimal medium A. Three drops of $2 \mu \mathrm{l}$ are deposited on glass slides containing a freshly made agarose pad (1X PBS $+1 \%$ agarose). Samples were observed with a Spinning disk (Yokogawa) W1 system mounted on a Zeiss inverted confocal microscope at 630-fold magnification. Using MicrobeJ (https://www.microbej.com/, (Ducret et al., 2016)), the position of aidB and fear loci is the cell in each condition at t0, t20, t40 and t60 after shift are calculated. An average of 600 cells were analyzed. Heat maps of the foci position are automatically generated by MicrobeJ. Two color localization was performed with gusC parS pMT1 (ter) and aidB parS $P 1$ locus (ori) tags. Cells were grown at $25^{\circ} \mathrm{C}$ in Minimal medium A supplemented with casaminoacids and glucose until culture reached $O D=0.1$ and then cells were shifted to $30^{\circ} \mathrm{C}$ for $1 \mathrm{~h}$. Cells were observed live on agarose pad on a thermo-controlled stage with a Zeiss inverted epifluorescence microscope equipped with led illumination. Localization of foci was recorded with the ObjectJ plugin of ImageJ https://sils.fnwi.uva.nl/bcb/objecti/. An average of 600 cells were analyzed.

\section{Acknowledgements}

We thank other members of the Espeli and Koszul groups, Sylvie Rimsky, Adrien Camus, Martial Marbouty, Théo Foutel Rodier for insightful discussions. Axel Cournac, Etienne Jean, Lyam Baudry, Vittore Scolari, Cyril Matthey Dorey, Remi Montagne for Computational analysis tips. We thank Ivan Matic, Arnaud Gutierrez and Wei Lin Su and Agnès Thierry for technical advices. We thank the CIRB 
bioRxiv preprint doi: https://doi org/10.1101/2021.05.17.444411; this version posted May 17, 2021. The copyright holder for this preprint

(which was not certified by peer review) is the author/funder, who has granted bioRxiv a license to display the preprint in perpetuity. It is made available under aCC-BY-NC-ND 4.0 International license.

658 imaging facility. We thank Jean-Yves Bouet, Pat Higgins, Frédéric Boccard and Ken Marians for strains

659 and plasmids. This research was supported by funding from the European Research Council under the

660 7th Framework Program (ERC Grant Agreement 771813 to R.K.) and ANR (HiResBac ANR-15-CE11-

661 0023-03 to R.K. and O.E).

662

\section{Author contributions}

BC, CC, RK and OE designed research. BC, IBC and CC performed the experiments, BC, IBC, CC

Declaration of interest

The authors declare no competing interests.

669

670

Data Availability

671 Sample description and raw sequences are accessible on SRA database through the following

672 accession number: PRJNA730396

673 


\section{References}

675

676

677

678

679

680

681

682

683

684

685

686

687

688

689

690

691

692

693

694

695

696

697

698

699

700

Badrinarayanan, A., Reyes-Lamothe, R., Uphoff, S., Leake, M.C., and Sherratt, D.J. (2012). In vivo architecture and action of bacterial structural maintenance of chromosome proteins. Science 338, 528-531.

Banigan, E.J., van den Berg, A.A., Brandão, H.B., Marko, J.F., and Mirny, L.A. (2020). Chromosome organization by one-sided and two-sided loop extrusion. ELife 9, e53558.

Baxter, J., Oliver, A.W., and Schalbetter, S.A. (2019). Are SMC Complexes Loop Extruding Factors? Linking Theory With Fact. BioEssays 41, 1800182.

Böhm, K., Giacomelli, G., Schmidt, A., Imhof, A., Koszul, R., Marbouty, M., and Bramkamp, M. (2020). Chromosome organization by a conserved condensin-ParB system in the actinobacterium Corynebacterium glutamicum. Nature Communications 11, 1485.

Carter, S.D., and Sjögren, C. (2012). The SMC complexes, DNA and chromosome topology: right or knot? Critical Reviews in Biochemistry and Molecular Biology 47, 1-16.

Cebrián, J., Castán, A., Martínez, V., Kadomatsu-Hermosa, M.J., Parra, C., Fernández-Nestosa, M.J., Schaerer, C., Hernández, P., Krimer, D.B., and Schvartzman, J.B. (2015). Direct Evidence for the Formation of Precatenanes during DNA Replication. J. Biol. Chem. 290, 13725-13735.

Champoux, J.J. (2001). DNA topoisomerases: structure, function, and mechanism. Annu Rev Biochem 70, 369-413.

Cockram, C., Thierry, A., Gorlas, A., Lestini, R., and Koszul, R. (2021). Euryarchaeal genomes are folded into SMC-dependent loops and domains, but lack transcription-mediated compartmentalization. Mol Cell 81, 459-472.e10.

Cournac, A., Marie-Nelly, H., Marbouty, M., Koszul, R., and Mozziconacci, J. (2012). Normalization of a chromosomal contact map. BMC Genomics 13, 436.

Cui, T., Moro-oka, N., Ohsumi, K., Kodama, K., Ohshima, T., Ogasawara, N., Mori, H., Wanner, B., Niki, H., and Horiuchi, T. (2007). Escherichia coli with a linear genome. EMBO Rep 8, 181-187.

Dame, R.T., Rashid, F.-Z.M., and Grainger, D.C. (2020). Chromosome organization in bacteria: mechanistic insights into genome structure and function. Nat Rev Genet 21, 227-242. 
701

702

703

704

705

706

707

708

709

710

711

712

713

714

715

716

717

Deng, S., Stein, R.A., and Higgins, N.P. (2005). Organization of supercoil domains and their reorganization by transcription. Mol Microbiol 57, 1511-1521.

DiGate, R.J., and Marians, K.J. (1989). Molecular cloning and DNA sequence analysis of Escherichia coli topB, the gene encoding topoisomerase III. J Biol Chem 264, 17924-17930.

Ducret, A., Quardokus, E.M., and Brun, Y.V. (2016). MicrobeJ, a tool for high throughput bacterial cell detection and quantitative analysis. Nature Microbiology 1, 1-7.

El Sayyed, H., Le Chat, L., Lebailly, E., Vickridge, E., Pages, C., Cornet, F., Cosentino Lagomarsino, M., and Espéli, O. (2016). Mapping Topoisomerase IV Binding and Activity Sites on the E. coli Genome. PLoS Genet 12.

Esnault, E., Valens, M., Espéli, O., and Boccard, F. (2007). Chromosome Structuring Limits Genome Plasticity in Escherichia coli. PLoS Genet 3, e226.

Espinosa, E., Paly, E., and Barre, F.-X. (2019). High-resolution whole-genome analysis of sisterchromatid cohesion. BioRxiv 2019.12.17.879379.

Ferullo, D.J., Cooper, D.L., Moore, H.R., and Lovett, S.T. (2009). Cell cycle synchronization of Escherichia coli using the stringent response, with fluorescence labeling assays for DNA content and replication. Methods $48,8-13$.

Ganji, M., Shaltiel, I.A., Bisht, S., Kim, E., Kalichava, A., Haering, C.H., and Dekker, C. (2018). Real-time imaging of DNA loop extrusion by condensin. Science 360, 102-105.

Grompone, G., Bidnenko, V., Ehrlich, S.D., and Michel, B. (2004). PriA Is Essential for Viability of the Escherichia coli Topoisomerase IV parE10(Ts) Mutant. J Bacteriol 186, 1197-1199.

Guo, M.S., Haakonsen, D.L., Zeng, W., Schumacher, M.A., and Laub, M.T. (2018). A bacterial chromosome structuring protein binds overtwisted DNA to stimulate type II topoisomerases and enable DNA replication. Cell 175, 583-597.e23.

Hayama, R., and Marians, K.J. (2010). Physical and functional interaction between the condensin MukB and the decatenase topoisomerase IV in Escherichia coli. PNAS 107, 18826-18831.

Hojgaard, A., Szerlong, H., Tabor, C., and Kuempel, P. (1999). Norfloxacin-induced DNA cleavage occurs at the dif resolvase locus in Escherichia coli and is the result of interaction with topoisomerase IV. Mol Microbiol 33, 1027-1036. 
Joshi, M.C., Magnan, D., Montminy, T.P., Lies, M., Stepankiw, N., and Bates, D. (2013). Regulation of Sister Chromosome Cohesion by the Replication Fork Tracking Protein SeqA. PLoS Genet 9.

Kato, J., Nishimura, Y., Imamura, R., Niki, H., Hiraga, S., and Suzuki, H. (1990). New topoisomerase essential for chromosome segregation in E. coli. Cell 63, 393-404.

Kavenoff, R., and Bowen, B.C. (1976). Electron microscopy of membrane-free folded chromosomes from Escherichia coli. Chromosoma 59, 89-101.

Kumar, R., Nurse, P., Bahng, S., Lee, C.M., and Marians, K.J. (2017). The MukB-topoisomerase IV interaction is required for proper chromosome compaction. J Biol Chem 292, 16921-16932.

Le, T.B., and Laub, M.T. (2016). Transcription rate and transcript length drive formation of chromosomal interaction domain boundaries. EMBO J. 35, 1582-1595.

Le, T.B.K., Imakaev, M.V., Mirny, L.A., and Laub, M.T. (2013). High-resolution mapping of the spatial organization of a bacterial chromosome. Science 342, 731-734.

Lee, C., and Marians, K.J. (2013). Characterization of the Nucleoid-associated Protein YejK. J Biol Chem 288, 31503-31516.

Leng, F., Chen, B., and Dunlap, D.D. (2011). Dividing a supercoiled DNA molecule into two independent topological domains. PNAS 108, 19973-19978.

Lesterlin, C., Gigant, E., Boccard, F., and Espéli, O. (2012). Sister chromatid interactions in bacteria revealed by a site-specific recombination assay. EMBO J 31, 3468-3479.

Levine, C., Hiasa, H., and Marians, K.J. (1998). DNA gyrase and topoisomerase IV: biochemical activities, physiological roles during chromosome replication, and drug sensitivities. Biochim Biophys Acta 1400, 29-43.

Li, Y., Stewart, N.K., Berger, A.J., Vos, S., Schoeffler, A.J., Berger, J.M., Chait, B.T., and Oakley, M.G. (2010). Escherichia coli condensin MukB stimulates topoisomerase IV activity by a direct physical interaction. PNAS 107, 18832-18837.

Lieberman-Aiden, E., van Berkum, N.L., Williams, L., Imakaev, M., Ragoczy, T., Telling, A., Amit, I., Lajoie, B.R., Sabo, P.J., Dorschner, M.O., et al. (2009). Comprehensive mapping of long-range interactions reveals folding principles of the human genome. Science 326, 289-293. 
756

757

758

759

760

761

762

763

764

765

766

767

768

769

770

771

772

773

774

775

776

777

778

779

780

781

782

783

Lioy, V.S., Cournac, A., Marbouty, M., Duigou, S., Mozziconacci, J., Espéli, O., Boccard, F., and Koszul, R. (2018). Multiscale Structuring of the E. coli Chromosome by Nucleoid-Associated and Condensin Proteins. Cell 172, 771-783.e18.

Luttinger, A.L., Springer, A.L., and Schmid, M.B. (1991). A cluster of genes that affects nucleoid segregation in Salmonella typhimurium. New Biol 3, 687-697.

Mäkelä, J., and Sherratt, D.J. (2020). Organization of the Escherichia coli Chromosome by a MukBEF Axial Core. Molecular Cell 78, 250-260.e5.

Marbouty, M., Le Gall, A., Cattoni, D.I., Cournac, A., Koh, A., Fiche, J.-B., Mozziconacci, J., Murray, H., Koszul, R., and Nollmann, M. (2015). Condensin- and Replication-Mediated Bacterial Chromosome Folding and Origin Condensation Revealed by Hi-C and Super-resolution Imaging. Molecular Cell 59, $588-602$.

Mariezcurrena, A., and Uhlmann, F. (2017). Observation of DNA intertwining along authentic budding yeast chromosomes. Genes Dev. 31, 2151-2161.

Marko, J.F., De Los Rios, P., Barducci, A., and Gruber, S. (2019). DNA-segment-capture model for loop extrusion by structural maintenance of chromosome (SMC) protein complexes. Nucleic Acids Res 47, 6956-6972.

McKie, S.J., Maxwell, A., and Neuman, K.C. (2020). Mapping DNA Topoisomerase Binding and Cleavage Genome Wide Using Next-Generation Sequencing Techniques. Genes 11, 92.

Mercier, R., Petit, M.-A., Schbath, S., Robin, S., El Karoui, M., Boccard, F., and Espéli, O. (2008). The MatP/matS site-specific system organizes the terminus region of the E. coli chromosome into a macrodomain. Cell 135, 475-485.

Nasmyth, K. (2001). Disseminating the genome: joining, resolving, and separating sister chromatids during mitosis and meiosis. Annu. Rev. Genet. 35, 673-745.

Nicolas, E., Upton, A.L., Uphoff, S., Henry, O., Badrinarayanan, A., and Sherratt, D. (2014). The SMC Complex MukBEF Recruits Topoisomerase IV to the Origin of Replication Region in Live Escherichia coli. MBio 5, e01001-13.

Nolivos, S., Upton, A.L., Badrinarayanan, A., Müller, J., Zawadzka, K., Wiktor, J., Gill, A., Arciszewska, L., Nicolas, E., and Sherratt, D. (2016). MatP regulates the coordinated action of topoisomerase IV and MukBEF in chromosome segregation. Nat Commun 7, 10466. 
785

786

787

788

789

790

791

792

793

794

795

796

797

798

799

800

801

802

803

804

805

806

807

808

809

810

811

812

Oomen, M.E., Hedger, A.K., Watts, J.K., and Dekker, J. (2020). Detecting chromatin interactions along and between sister chromatids with SisterC (Genomics).

Orr, E., and Staudenbauer, W.L. (1981). An Escherichia coli mutant thermosensitive in the B subunit of DNA gyrase: Effect on the structure and replication of the colicin E1 plasmid in vitro. Mol Gen Genet 181, 52-56.

Peter, B.J., Arsuaga, J., Breier, A.M., Khodursky, A.B., Brown, P.O., and Cozzarelli, N.R. (2004).

Genomic transcriptional response to loss of chromosomal supercoiling in Escherichia coli. Genome Biology 5, R87.

Postow, L. (2004). Topological domain structure of the Escherichia coli chromosome. Genes \& Development 18, 1766-1779.

Ruiten, M.S. van, and Rowland, B.D. (2018). SMC Complexes: Universal DNA Looping Machines with Distinct Regulators. Trends in Genetics 34, 477-487.

Sawitzke, J.A., and Austin, S. (2000). Suppression of chromosome segregation defects of Escherichia coli muk mutants by mutations in topoisomerase I. Proc Natl Acad Sci U S A 97, 1671-1676.

Sharma, U.K., and Chatterji, D. (2010). Transcriptional switching in Escherichia coli during stress and starvation by modulation of sigma activity. FEMS Microbiol Rev 34, 646-657.

Sinden, R.R., and Pettijohn, D.E. (1981). Chromosomes in living Escherichia coli cells are segregated into domains of supercoiling. Proc Natl Acad Sci U S A 78, 224-228.

Springer, A.L., and Schimd, M.B. (1993). Molecular characterization of the Salmonella typhimurium parE gene. Nucl Acids Res 21, 1805-1809.

Staczek, P., and Higgins, N.P. (1998). Gyrase and Topo IV modulate chromosome domain size in vivo. Mol Microbiol 29, 1435-1448.

Stein, R.A., Deng, S., and Higgins, N.P. (2005). Measuring chromosome dynamics on different time scales using resolvases with varying half-lives: Detecting domains with time-restricted enzymes. Molecular Microbiology 56, 1049-1061.

Stracy, M., Ginda, K., Zawadzka, K., Lesterlin, C., Kapanidis, A.N., and Sherratt, D.J. (2015). The Localization and Action of Topoisomerase IV in Escherichia coli Chromosome Segregation Is Coordinated by the SMC Complex, MukBEF. Cell Rep 13, 2587-2596. 
bioRxiv preprint doi: https://doi org/10.1101/2021.05.17.444411; this version posted May 17, 2021. The copyright holder for this preprint

(which was not certified by peer review) is the author/funder, who has granted bioRxiv a license to display the preprint in perpetuity. It is made available under aCC-BY-NC-ND 4.0 International license.

813 Subramanian, D., Kraut, E., Staubus, A., Young, D.C., and Muller, M.T. (1995). Analysis of

814 topoisomerase I/DNA complexes in patients administered topotecan. Cancer Res 55, 2097-2103.

815 Val, M.-E., Marbouty, M., Martins, F. de L., Kennedy, S.P., Kemble, H., Bland, M.J., Possoz, C., Koszul,

816 R., Skovgaard, O., and Mazel, D. (2016). A checkpoint control orchestrates the replication of the two

817 chromosomes of Vibrio cholerae. Science Advances 2, e1501914.

818 Wang, X., Reyes-Lamothe, R., and Sherratt, D.J. (2008). Modulation of Escherichia coli sister

819 chromosome cohesion by topoisomerase IV. Genes Dev 22, 2426-2433.

820 Wang, X., Le, T.B.K., Lajoie, B.R., Dekker, J., Laub, M.T., and Rudner, D.Z. (2015). Condensin promotes

821 the juxtaposition of DNA flanking its loading site in Bacillus subtilis. Genes Dev. 29, 1661-1675.

822 Zechiedrich, E.L., Khodursky, A.B., and Cozzarelli, N.R. (1997). Topoisomerase IV, not gyrase,

823 decatenates products of site-specific recombination in Escherichia coli. Genes Dev 11, $2580-2592$. 